Comme ce couplage ne portera que sur les stators, il sera extrêmement facile à réaliser.

3o Freinage. - On peut utiliser une propriété particulière de ce moteur pour remplacer le freinage électrique réalisé avec le moteur-série à courant continu.

Intercalons une résistance ohmique $r$ dans le rotor et faisons $\theta=0$ dans les égalités (3) et (4); elles deviennent :

$$
E=L \frac{d I}{d t}
$$

$$
M \Omega I=l \frac{d i}{d t}+r i
$$

d'où : $\quad I_{\text {eff }}=\frac{E_{\text {eff }}}{L \omega}\left(5^{\prime}\right)$

$$
i_{\mathrm{eff}}=\frac{E_{\mathrm{eff}}}{L \omega} \times \frac{M \Omega}{\sqrt{r^{2}+l^{2} \omega^{2}}}
$$

Dans ce cas, le moteur fait frein et le couple résistant $\Gamma$ est donné évidemment par les égalités suivantes qui expriment que la puissance mécanique absorbée est employée à chauffer la résistanee $r: r i^{2}{ }_{\mathrm{eff}}=\Gamma \Omega$, d'où, en remplaçant $i_{\text {eff }}$ par sa valeur :

$$
\Gamma=\frac{M^{2} E^{2} \mathrm{eff}}{L^{2} \omega^{2}} \times \frac{r}{r^{2}+l^{2} \omega^{2}} \Omega
$$

expression analogue à celle qui donne le couple résistant d'un moteur en dérivation dont l'excitation est alimentée par le réseau et dont l'induit est fermé sur une résistance. Toutefois, pour obtenir le maximum d'effet, il ne faut pas, dans le cas présent, que la résistance $r$ soit négligeable, mais qu'elle satisfasse à la relation : $r=\omega l$.

Pendant le freinage $I_{\text {en }}$ reste constant et garde une faible valeur donnée par (5').

Le réseau ne fournit que la puissance dépensée en chaleur par le passage du courant $I_{\text {eff }}$ dans la résistance du stator.

Pour exciter le stator, on pourrait aussi employer le courant fourni par une petite batterie d'accumulateurs ou une dynamo auxiliaire de faible puissance. Ce dernier procédé a l'avantage de garder son efficacité dans le cas d'une rupture de contact entre la voiture et la ligne.

Dans l'un et l'autre cas le courant peut servir à actionner des freins électromagnétiques. On a ainsi un frein sûr et facilement réglable.

$4^{0}$ Emplor de hautes tensions. - Le stator étant seul alimenté par le réseau, il serait facile d'utiliser directement le courant à haute tension, sans transformateurs.

Conclusions. - Ces quelques considérations montrent donc que ce moteur aurait de sérjeux avantages pour la traction en particulier, et qu'il mériterait une étude et des essais sérieux. Le point le plus délicat à étudier semble devoir être la commutation, mais nous croyons qu'on peut arriver à la réaliser dans des conditions suffisantes.

\section{Joseph Bethenod,} Ancien élève de l'Ecole Centrale Lyonnaise.

\section{NOTES ANNEXES}

Note $I_{1}-$ On a, d'après les lois générales de l'induction :

$$
e=\frac{d(m I)}{d t}=m \frac{d I}{d t}+I \frac{d m}{d t}
$$

$$
\begin{array}{lc}
\text { Or, } & m=M \sin \theta \\
\text { Donc : } & e=M \sin \theta \frac{d I}{d t}+M I \cos \theta \times \frac{d 0}{d t}
\end{array}
$$

Il est évident que $: \frac{d \theta}{d t}=\mp \Omega$ suivant le sens de rotation par rapport au calage des balais.

On a bien finalement : $e=M \sin \theta \frac{d I}{d t} \mp M \Omega \cos \theta \times I$.

Note II. - Si on pose $\frac{M^{2}}{L l}=u<\mathrm{I}$ tout revient à rendre l'expression $x=\frac{\mathrm{I}-u \sin ^{2} \theta}{u \sin \theta \cos \theta}$ minimum.

Or, on a: $\sin ^{2} \theta=\frac{1-\cos 2 \theta}{2}, \quad \sin \theta \cos \theta=\frac{\sin 2 \theta}{2}$

Donc: $\quad x=\frac{2-u+u \cos 2 \theta}{u \sin 2 \theta}=\frac{2-u}{u} \cdot \frac{\mathrm{I}}{\sin 2 \theta}+\frac{1}{\operatorname{tg} 2 \theta}$

En annulant la dérivée de $x$ par rapport à $2 \theta$, on a :

$-\frac{2-u}{u} \frac{\cos 2 \theta}{\sin ^{2} 2 \theta}-\frac{1}{\cos ^{2} 2 \theta} \times \frac{\cos ^{2} 2 \theta}{\sin ^{2} 2 \theta}=0 \quad \cos 2 \theta=\frac{-u}{2-u}$

Note III. - On a : $\quad P=E_{\text {eff }} I_{\text {eff }} \cos \%$

$$
E_{\mathrm{eff}} \sin \varphi=\frac{L l-M^{2} \sin \theta}{l} \omega \times I_{\mathrm{eff}}
$$

d'où on tire : $P=E^{2}{ }_{\text {eff }} \times \frac{l}{\left(L l-M^{2} \sin ^{2} \theta\right) \omega} \times \sin \varphi \cos \varphi$, pour un angle $\theta$ donné et une fréquence déterminée:

$$
\begin{aligned}
& \frac{E_{\mathrm{eff}}^{2} \times l}{\left(L l-M^{2} \sin ^{2} \theta\right) \omega}=\text { constante }=W . \\
& P=W \times \sin \varphi \times \cos \varphi=W \times \frac{\sin 2 \varphi}{2}
\end{aligned}
$$

Donc le maximum de $P$ a bien lieu pour $\varphi=\frac{\pi}{4}$.

Vérification expérimentale. - Il est facile de se rendre compte, au moins qualitativement, du fonctionnement de cet alternomoteur. On n'a qu'à prendre une dynamo à courant continu, à inducteur feuilleté de préférence, et à lancer un courant alternatif dans l'inducteur, après avoir court-circuité les balais: l'induit se met à tourner suivant le sens du décalage des balais et, même dans cet essai rudimentaire, on constate que le couple moteur est maximum au démarrage.

J. B.

\section{EUPLOI DES COURANTS TRIPHASÉS A HAUTE TENSION}

\author{
pour la traction électriquo sur los grandes lignes
}

\section{APPLiGation A LA LIGNE DE LA VALTELINE}

Jusqu'à ces dernières années, l'emploi des courants continus à 500-700 volts a été à peu près général dans les applications de la traction électrique. Ce choix était d'ailleurs indiqué pour les réseaux de tramways urbains oủ la puissance des moteurs varie de 15 à 50 chevaux, où le poids des voitures est d'environ ro tonnes et les distances parcourues de 10 à 20 kilomètres, avec des démarrages fréquents et des vitesses faibles.

Tout autres sont les conditions qui se présentent pour 
l'exploitation électrique des grandes lignes de chemins de fer. Il s'agit alors de puissances de 200 à 1200 chevaux, de poids considérables à remorquer, de parcours de plusieurs centaines de kilomètres, de grandes vitesses et de trains se succédant ą̀intervalles de temps éloignés.

Un exemple très intéressant de traction électrique se présentant dans ces conditions a été inauguré récemment par l'Etat Italien sur les lignes de la Valteline. Nous devons à l'obligeance de la Maison Ganz et $\mathrm{Co}$, de Budapest, qui a étudié et si heureusement résolu cette délicate question, les quelques considérations et descriptions suivantes, qu'elle nous a autorisé à reproduire. Notons aussi que M. Korda, dans une des dernières séances de la Société Internationale des Electriciens, a déjà traité ce même sujet.

\section{CONSIDÉRATIONS GÉNÉRALES}

Le rapport entre la charge moyenne et la charge maxima dans les stations centrales de tramways, sur lesquelles les trains ou voitures se succèdent à intervalles de 5 à $20 \mathrm{mi-}$ nutes, ne varie que de I à 3 . Si l'on fait par contre un calcul approximatif pour de grandes lignes, le rapport considéré sera de beaucoup plus défavorable.

Considérons une ligne en palier de 15 kil. de longueur (longueur maxima qui peut être desservie rationnellement par une sous-station), dont les gares se trouvent écartées de 8 kil. Les trains se succèdent à des intervalles de $\mathrm{I}$ h. 30 dans chaque sens. Soit 70 kilomètres à l'heure la vitesse maxima, et 300 tonnes le poids d'un train. Supposons que nous ayons établi l'horaire de manière que deux trains ne démarrent jamais simultanément. La charge maxima est alors produite par un train en démar-

rage et un train en route. Dans ce cas, la charge maxima sera 7 fois plus grande que la charge moyenne.

Si nous permettons le démarrage simultané de deux trains, ou si trois trains se trouvent à la fois en mouvement, le rapport considéré atteint le chiffre de so. Il ne faut pas cependant oublier que les données se rapportent à un trafic relativement intense. Avec un trafic moins intense, ce rapport croît rapidement. Si nous étions obligés de proportionner les stations ou sous-stations de manière à pouvoir répondre à une surcharge momentanée d'une telle valeur, leur rendement moyen serait mauvais, les frais d'installation seraient élevés, et la traction électrique cesserait d'être lucrative. Dans ce cas nous sommes forcément obligés de donner à ce système rigide l'élasticité qu'il exige. L'emploi des accumulateurs s'impose par lui-même. Mais les frais d'installation, et surtout ceux d'entretien, abaissent considérablement la valeur pratique d'une telle solution.

Une batterie d'accumulateurs, calculée pour l'exemple précédent, coûterait environ 170.000 francs, avec une dépense annuelle de 7 à 9 mille francs pour l'entretien.

Si nous remplaçons les sous-stations nécessaires dans le système à courant continu par des transformateurs qui réduisent convenablement la tension primaire, et si nous alimentons directement les conducteurs de contact par le courant polyphasé secondaire, la solution devient beaucoup moins coûteuse.
Nous savons quela surcharge d'un transformateur à courant alternatif n'est limitée que par l'élévation de la tempé. rature ou par la chute de potentiel. Les dynamos is courant continu supportent difficilement une surcharge de $50 \%$.

Si c'est l'élévation de température, et non la chute de potentiel qui limite les dimensions d'un transformateur, on peut se rendre compte que, dans l'exemple précédent, il faudrait une dynamo à courant continu d'une puissance normale d'au moins $1.000 \mathrm{kwatts}$, tandis qu'un transformateur de 350 kwatts suffit dans le cas de courants alternatifs.

Il en résulte qu'une machine à courant continu, desservant la ligne précédente, devrait avoir des dimensions trois fois plus grandes que celles qu'exigerait la charge moyenne sans l'emploi d'accumulateurs; tandis qu'un transformateur à courants alternatifs dont les dimensions dépasseraient d'environ $67 \%$ celles qui correspondent à la charge moyenne, suffit pour.le service.

Un transformateur pareil coûterait environ 25.000 francs, c'est à-dire à peu près la sixième partie du prix de la batterie d'accumulateurs, auquel on devrait encore ajouter le prix de la génératrice ou commutatrice.

Cette comparaison devient encore beaucoup plus favorable pour les courants alternatifs, si nous élevons la

tension dans les fils de contact. Avec cette élévation, la longueur d'un tronçon de ligne, desservi par un transformateur, croît aussi, ce qui correspond à une augmentation de sa charge moyenne. Par conséquent, le rapport entre la charge maxima et la charge moyenne diminue, et l'exploitation devient plus économique en même temps que la dépense d'installation diminue.

Cette conclusion s'applique encore mieux aux grandes stations centrales, alimentant les sous-stations. En effet, le rayon desservi par une seule station centrale croît avec l'augmentation de la tension primaire, par suite le nombre des sous-stations desservies par une seule centrale peut aussi augmenter, de sorte que les inégalités de charges dans les divers tronçons peuvent, en partie du moins, se compenser aux bornes du tableau de distribution. Le rapport entre les charges maxima et moyenne devient encore plus avantageux.

Un autre avantage de l'augmentation de la tension consiste dans l'économic réalisée sur le cuivre employé pour les conducteurs d'alimentation.

Les sections des conducteurs sont proportionnelles aux 
carrés des courants. En comparant la quantité de cuivre nécessaire avec courants continus à 700 volts, et avec courants triphasés à 3.000 volts, on trouve qu'elle est 10 fois plus grande dans le premier cas que dans le second, à égalité de distance, d'énergie transmise et de perte de tension.
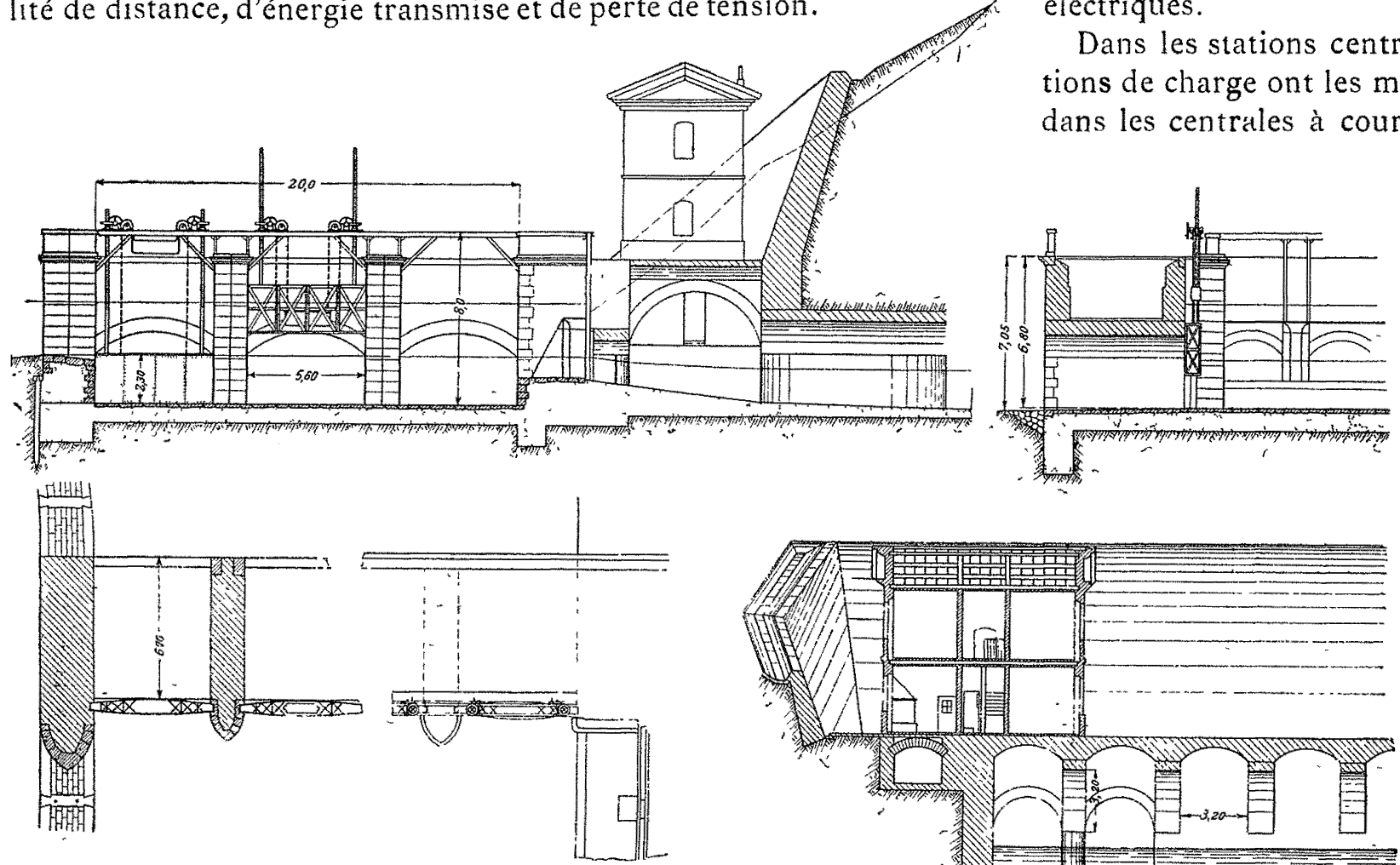

Fig. 2. - Plañs du barrage et de la prise d'eau.

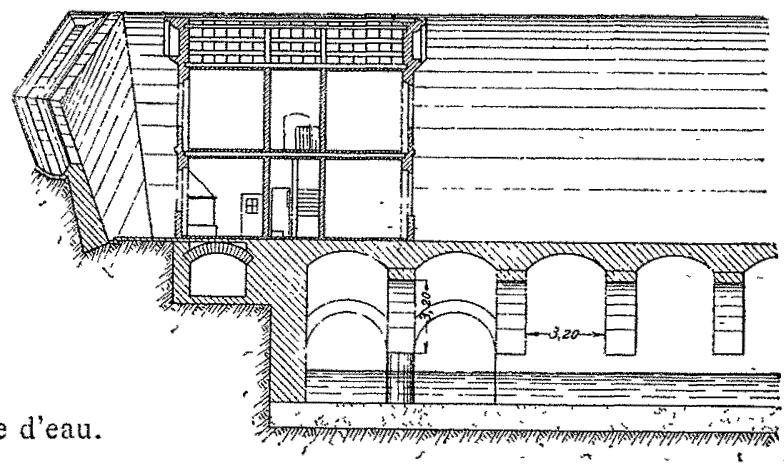

c'est-à-dire 20.000 francs par kil. Si nous remplaçons ce conducteur par un troisième rail, celui-ci pèserait environ $62^{\mathrm{k}} 5$ par mètre à équivalence de conductibilité. Le prix de ce dernier est encore de 14.000 francs, sans les éclissages électriques.

Dans les stations centrales, les grandes variations de charge ont les mêmes inconvénients que défavorable sur l'éco. nomie de l'exploita. tion et sur la dépense d'installation. Là tension primaire doit donc avoir une valeur telle, que les sousstations de la ligne entière puissent être alimentées d'une śeule centrale, car les variations de charge des tronçons se compensent en partie aux bor* nes du tableau de dis. tribution et le rap. port entre la charge maxima et la charge moyenne décroît.

On peut done affir.

Ce qui revient à dire que pour une même section totale de conducteur, la distance entre les sous-stations voisines est 10 fois plus grande dans le cas des courants alternatifs à 3.000 volts, que dans le cas des courants continus à 700 volts, les autres conditions restant les mêmes.

Dans cette comparaison nous avons négligé la perte d'énergie dans les rails, aússi bien dans un cas que dans l'autre. Il convient cependant de remarquer que la perte dans les conducteurs de retours (rails) est d'autant mains négligeable que l'intensité du courant est plus grande. Dans le cas de haute tension et petite intensité, la perte dans les rails est négligeable relativement aux pertes dans les conducteurs, mais non pas dans le cas des courants continus à 500-700 volts, si l'énergie transmise atteint 500-I .000 chevaux. Faisant entrer certe perte dans notre calcul comparatif de la quantité de cuivre nécessaire, on trouve encore de meilleurs résultats en faveur de la haute tension.

Le coût des conducteurs dé contact, pour

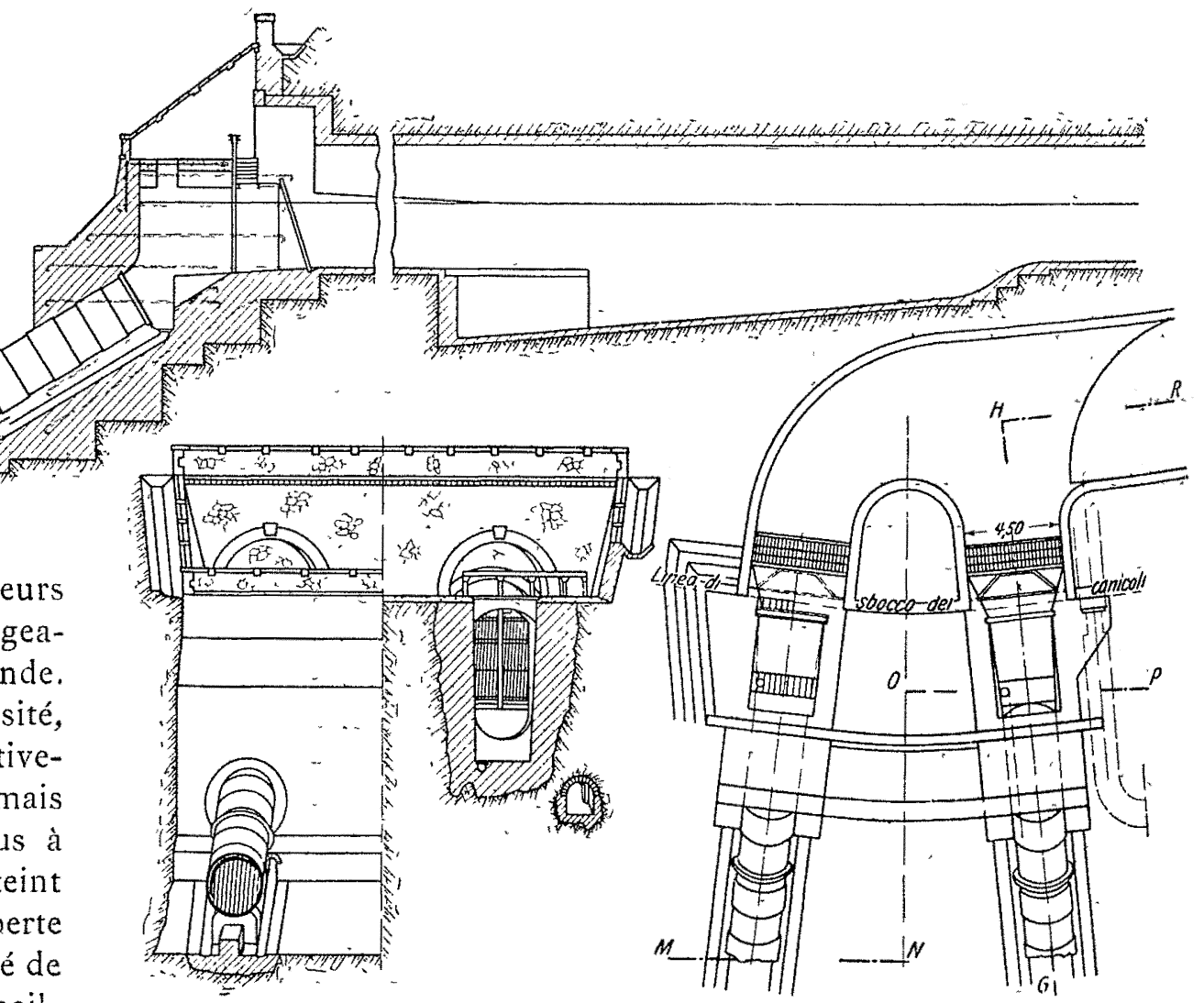

Fig. 3, - Chambre de décantation et départ des conduites forcées,

des courants polyphasés à 3.000 volts, admettant deux fils de $8 \mathrm{~m} / \mathrm{m}$ de diamètre, monte environ à. 2.000 francs par kil. La section correspondante pour. des courants continus, et partant le prix des conducteurs seront 10 fois plus grands,

mer que l'augmentation de la tension dans les fils de contact jusqu'à la limite extrême n'est pas motivée, tandis que celle du courant primaire aux bornes du tableau de distribution peut et doit être la plus grande possible. 
La tension secondaire est encore déterminée par une autre circonstance, à savoir : par l'intensité du courant qui s'écoule du conducteur à travers l'appareil de prise de courant.

Lorsqu'on emploie comme conducteur d'amenée un troisième rail, l'intensité du courant à capter ne donne lieu à aucun inconvénient, car si elle est trop grande pour pouvoir être supportée par une seule prise de courant, rien n'empêche de disposer plusieurs sabots. Mais si l'alimentation se fait avec des conducteurs aériens, cette ressource fait défaut. d'intensité pouvant être prise des conducteurs aériens, l'énergie maxima transmise aux moteurs sera en fonction du voltage appliqué.

A ce point de vue donc, une tension de 3.000 volts, qui correspond à une puissance de 1.500 chevaux, suffit pour pouvoir remorquer un train du poids adopté aujourd'hui sur nos grandes lignes interurbaines.

Jusqu'ici, nous avons déterminé la limite rationnelle supérieure de la tension à appliquer. Quels sont maintenant les désavantages d'une tension élevée au-delà de cette limite? Les désavantages principaux consistent dans

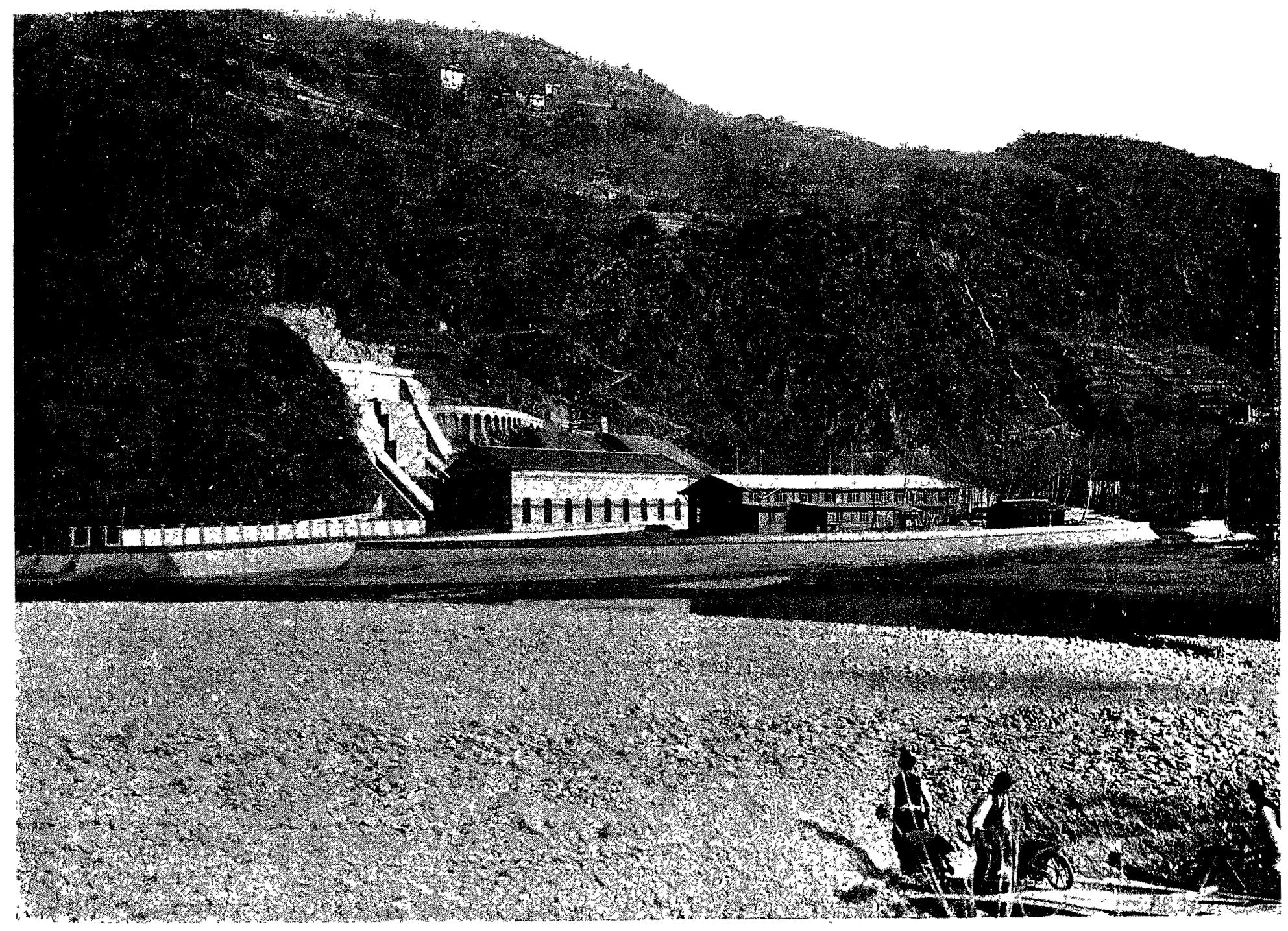

Fig. 4. - Vue d'ensemble de la station centrale génératrice.

La pression des trolleys ou des archets contre les fils de contact ne doit pas, en effet, compenser entièrement le poids de ceux-ci, car une oscillation dangereuse de ces fils en pourrait être la conséquence.

L'intensité maxima du courant pouvant être captée par un contact roulant d'un fil aérien est, suivant l'observation de M. de Kando, lors de son voyage d'étude en Amérique, 300 ampères, avec fonctionnement parfait du trolley, observation faite à plusicurs reprises sur la ligne Détroit-MontClément. Il a constaté aussi des prises jusqu'à 500 ampères sur la ligne New-York-New-Haven-Hartford, mais le trolley était sujet, dans ce cas, à une usure rapide.

Si nous posons donc 300 ampères comme maximum les difficultés d'isolation et de construction, tant du moteur que de tous les appareils servant à la réception ou manipulation du courant.

La tension du courant dans les fils de contact sur la ligne de Marienfeld à Zossen, de la Société berlinoise « Studiengesellschaft " était de 12.000 volts. Au cours des essais effectués, qui servaient principalement à déterminer la limite supérieure de la vitesse d'un train, remorqué électri. quement, cette tension de contact fut abaissée dans la voiture de l'Allgemeine-Electricitats-Gesellschaft, à-435, dans la voiture de la Société Siemens et Halske à 1. . 50 volts (pendant le démarrage à 1.850 ), et le courant fut amené sous cette tension aux moteurs. 
Mais en faisant cette transformation, nous sacrifions un des plus grands avantages de la traction électrique : la légèreté des voitures motrices, car le poids du transformateur nécessaire augmente avec la puissance du véhicule moteur. Un transformateur calculé pour une locomotive de 1.200 à I .300 chevaux pèserait environ i 5 tonnes.

Il n'est donc pas pratique d'augmenter la tension dans les fils de contact, moyennant un pareil prix.Il vaut alors mieux admettre, comme limite pratique, la tension qui permet la construction rationnelle des moteurs et des autres appareils électriques, et qui assure un bon fonctionnement de l'appareil de prise de courant, dans les circonstances de la traction d'aujourd'hui.

La principale objection contre l'application des courants à haute tension pourla traction était le danger auquel le personnel et les voyageurs sont exposés, en cas d'un contact avec les conducteurs.

Cette difficulté a été très élégamment résolue par la maison Ganz, de la manière suivante : au lieu de chercher la sécurité à l'aide d'isolants de plus en plus épais et parfaits,

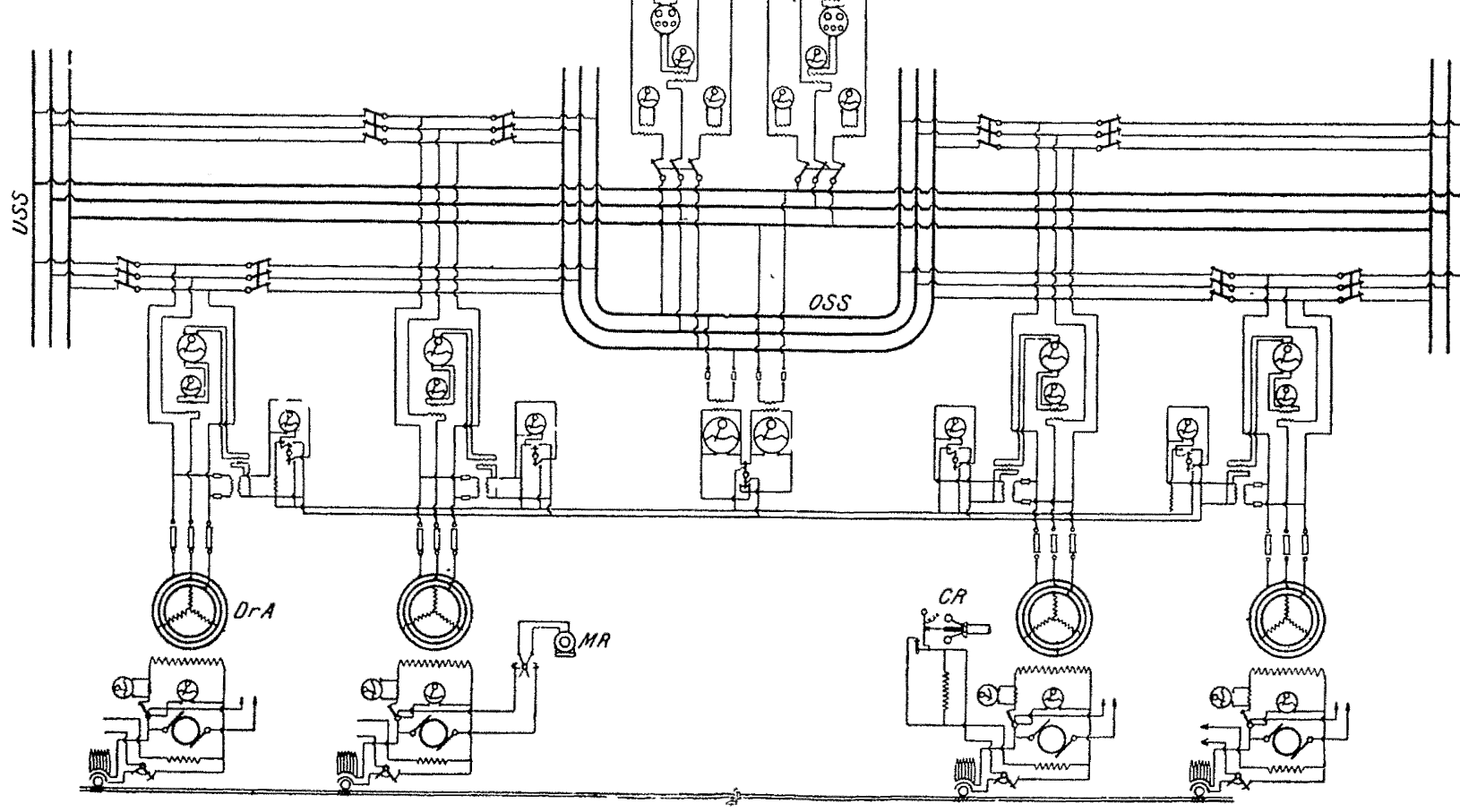

Fig. 6. - Schéma des connexions de l'usine génératrice.

DrA, Généraurce triphasée - $M R$, Noteur du régulateur de la turbine - $C h$, Régulateur centrifuge (Nombre de tours normal : 150 ; nombre de tours masmum: 170). - USS, Barres collectrices supérneures. - USS, Barres collectrices inférieures. - $L$, Plaques connectées à la terre. et qui n'en étaient pas moins sujets à des détériorations, et par suite à des accidents, on a songé à envelopper simpleenveloppe métallique continue, et de mettre cette enveloppe en communication avec le truc de la voiture, et partant, par l'intermédiaire des roues, avec les rails et la terre. ment tout appareil et conducteur à haute tension d'une
L'enveloppe métallique qui n'intervient, d'ailleurs, que s'il y a un défaut dans l'isolation, joue ainsi le rôle d'un parafoudre, en amenant le courant à la terre.

Une rupture accidentelle du conducteur aérien, qui vient tomber sur le toit de la voiture, devient alors sans danger

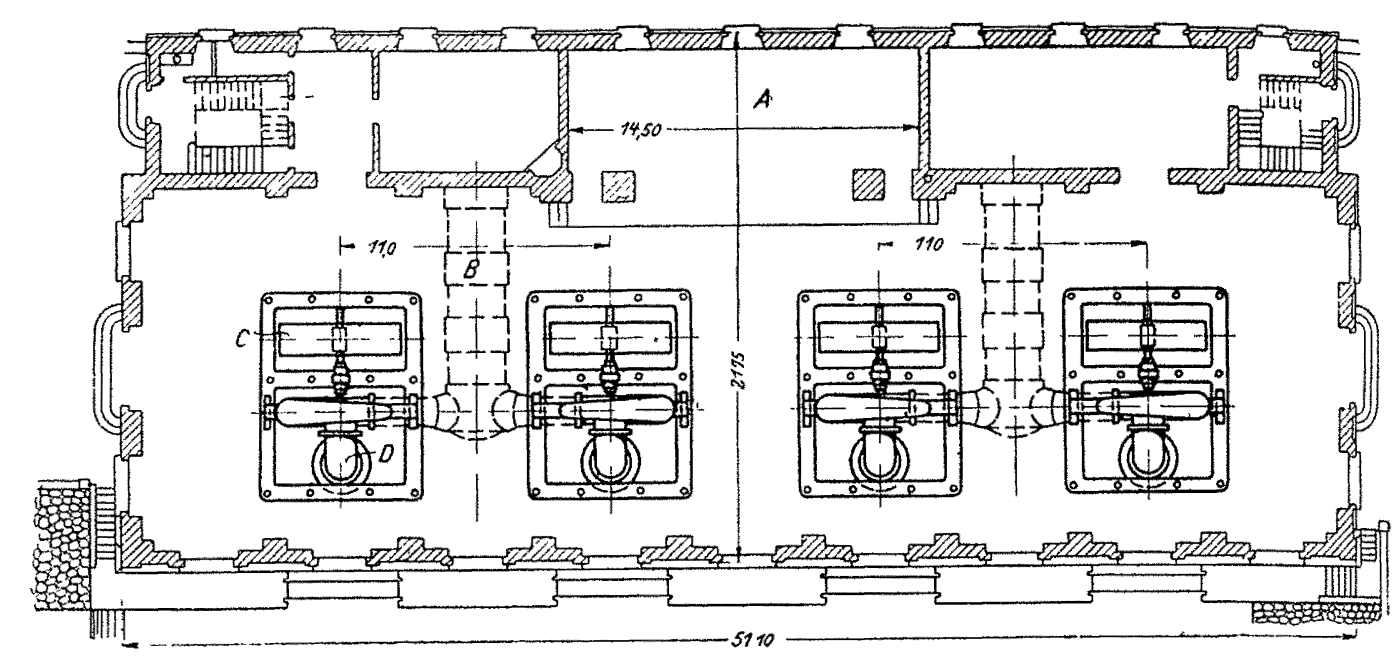

Fig. 5. - Plan de la salle des machines de la station centrale génératrice. 
important, en cas d'une réparation quelconque des conduc. teurs ou des transformateurs.

Mais cette objection ne s'applique pas aux conducteurs primaires alimentant les sous-stations.

Ceux-ci ne supportant aucun frottement mécanique sont, en effet, moins sujets à rupture que les fils de contact.

Le désavantage des courtes sections, c'est-à-dire le rapport défavorable entre charges moyenne et maxima, ne se fait pas sentir à un degré aussi nuisible dans les sous-sta-
En effet, si un train descend une pente et, si par suite de la gravité, sa vitesse augmente au-delà de la vitesse de régime, le train est automatiquement freiné par ses moteurs. Mais l'énergie de la force vive n'est pas transformée en chaleur par les sabots des freins ordınaires; elle est récupérée sous forme de courants électriques et renvoyée aux conducieurs.

Le couplage des moteurs en cascade permet la diminution de la vitesse de régime à la moitié de sa valeur normale; on peut marcher à deux vitesses différentes. Si lorsqu'un

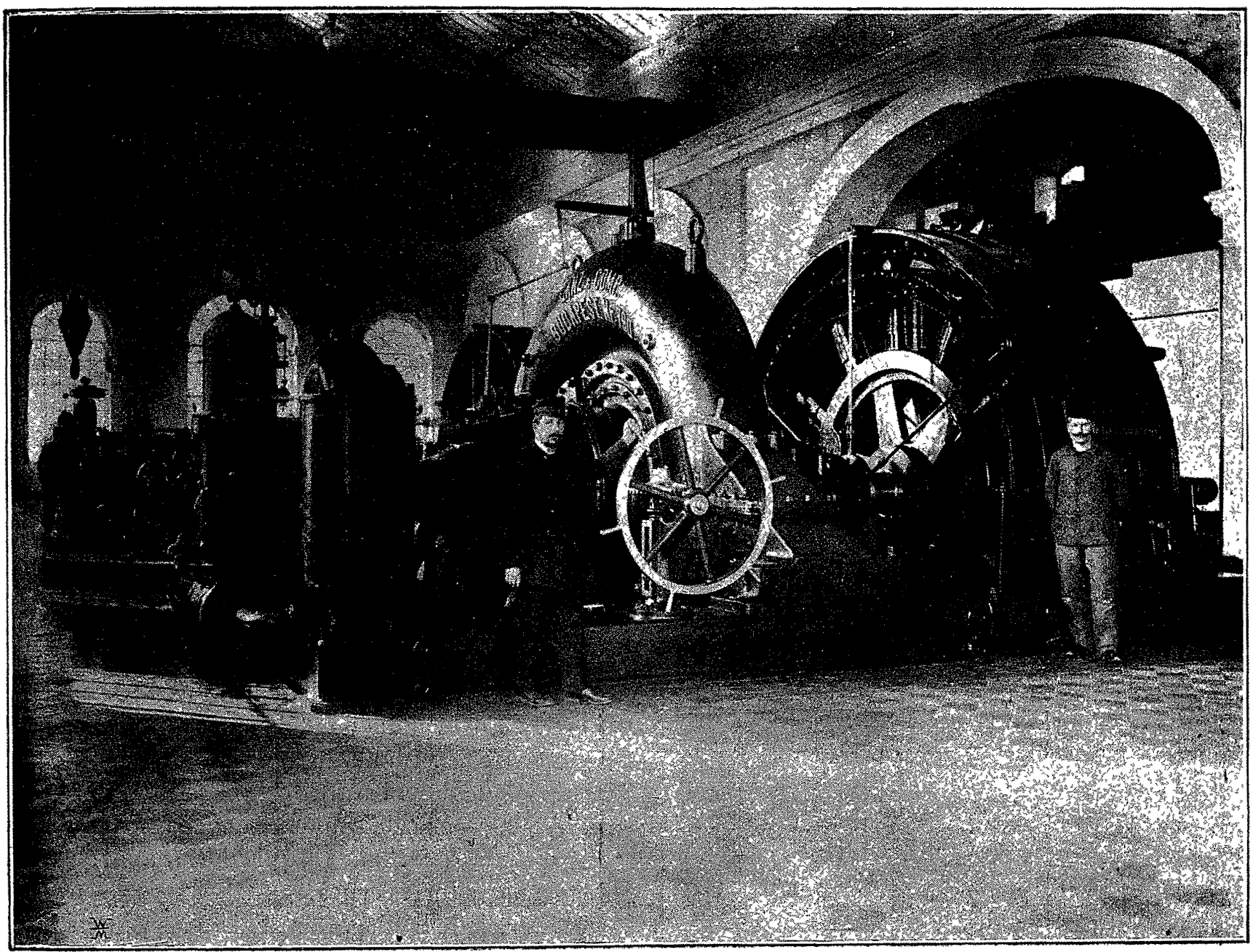

Fig. 7. - Vue intérieure de la station centrale génératrice.

tions à transformateurs statiques que dans les sous-stations à transformateurs rotatifs.

Si ce rapport ne dépasse pas le chiffre de 5-6, il est inutile d'élever la tension pour accroître la longueur d'une section, car nous avons vu qu'un transformateur supporte facilement une charge momentanée égale à cinq ou six fois sa charge normale sans grand inconvénient.

L'application des courants triphasés assure encore un avantage considérable provenant de la qualité des moteurs qui, en les faisant tourner par une source extérieure au-delà de la vitesse correspondant au synchronisme, deviennent générateurs et restituent de l'énergie à la ligne.

Cette particularité des moteurs polyphasés devient très précieuse pour l'exploitation électrique des grandes lignes. train marche à pleine vitesse, on couple les moteurs en cascade, la force vive correspondant à la différence des deux vitesses est également récupérée sous forme d'énergie électrique, et la vitesse du train est réduite à moitié, sans aucun freinage mécanique.

\section{DESCRIPTION GÉNÉRALE DE L'INSTALLATION}

Le chemin de fer qui suit la vallée de l'Adda et le bord du lac de Como se partage en trois lignes, celles de :

Lecco-Colico, d'une longueur de..... 38,95 kil.

Colico-Sondrio, d'une longueur de... 40,79 "

Colico-Chiavenna, d'une longueur de.

En somme........ ı. o6,3 r kil. 
Le trafic des marchandises est assez intense sur cette ligne, mais il est encore surpassé par celui des passagers, surtout en été, lorsque les touristes arrivant de Splugen et de l'Engadine par la diligence à Chiavenna et de la Bernina à Sondrio, continuent leur voyage sur cette ligne vers la Lombardie.

Fig. 8. - Poteau à double potence de la ligne secondaire.

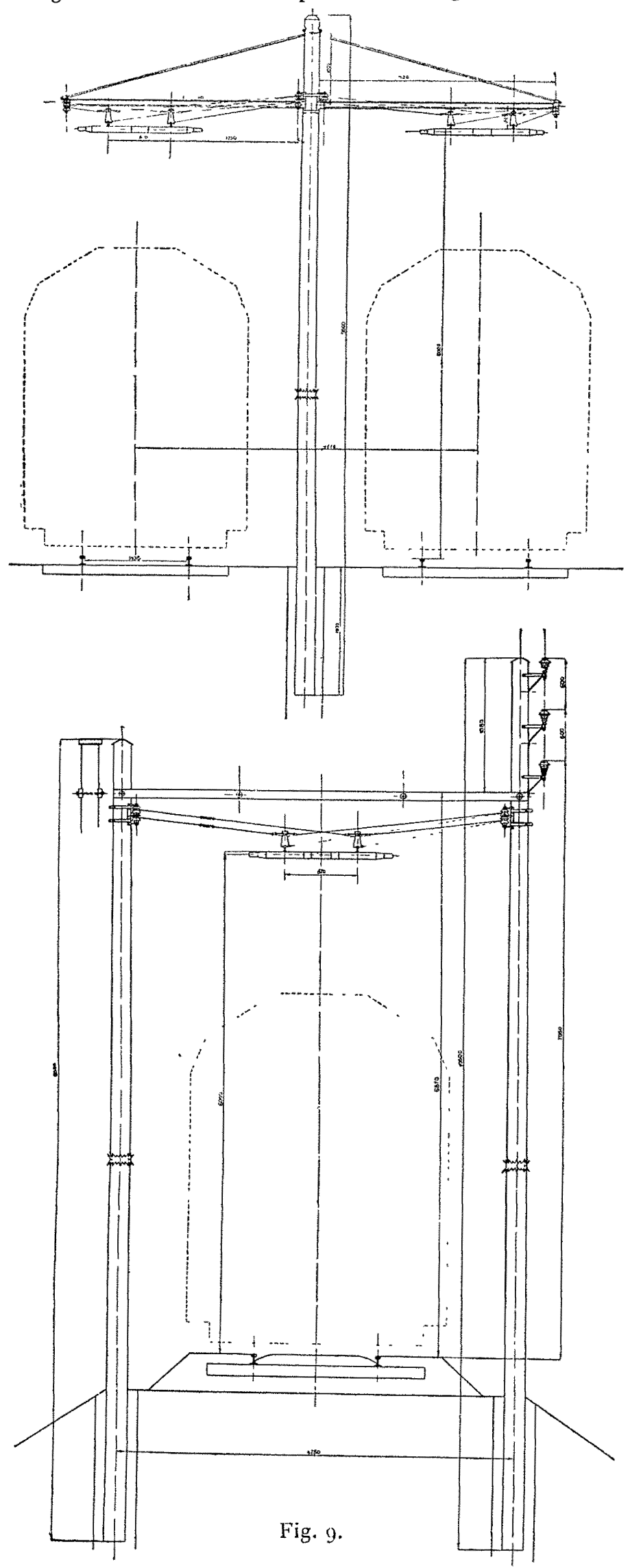

Poteaux doubles supportant les lignes primaire et secondaire.
L'énergie nécessaire pour le trafic électrique de la Valteline est fournie par la station centrale près de Morbegno.

Le courant triphasé à 20.000 volts y est directement produit aux bornes des générateurs et conduit par la ligne primaire aux sous-stations, distribuées le long de la voie. Les transformateurs statiques réduisent la tension primaire de 20.000 volts à 3.000 volts, et alimentent directement la ligne de contact. Le courant de 3.000 volts traverse l'appareil de prise de courant et entre directement dans les inducteurs des moteurs.

$1^{\circ}$ Station centrale. - L'Adda, qui fournit l'énergie nécessaire à la station centrale, a une chute d'environ 35 mètres sur une longueur de 5 kilomètres entre les ponts de Desco et de Ganda. Son bassin de réception est de 2.550 kilomètres carrés et la minimum du débit est $25 \mathrm{~m}^{3}$.

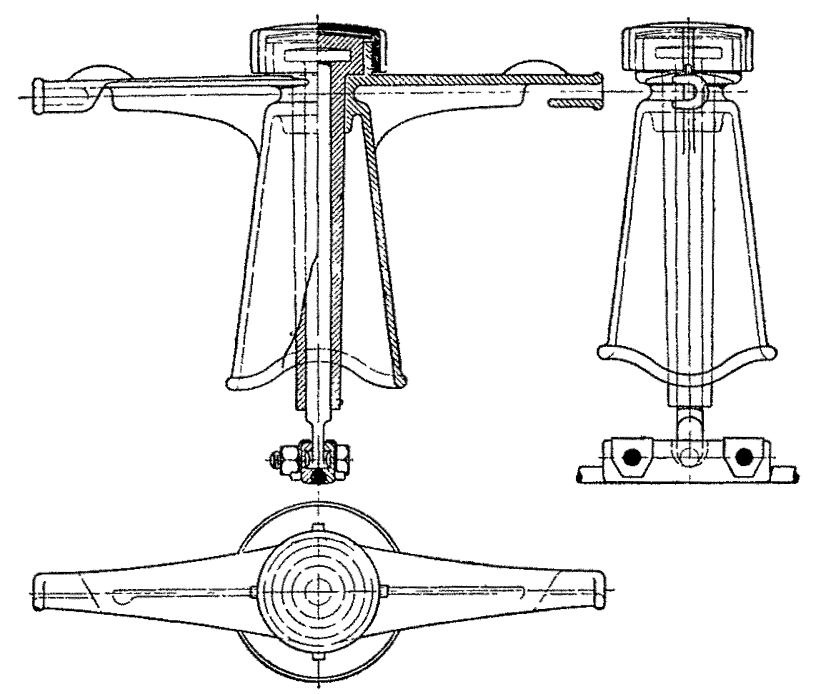

Fig. ro. - Isolateur en ambroine.

L'énergie minima disponible est de 7.500 chevaux effectifs. En aval du pont de Desco le fleuve se partage en deux branches. L'une des deux a été définitivement barrée pendant que dans l'autre on a ménagé un barrage de retenue mobile composé de vannes à soulever pour faire évacuer les apports de l'Adda, qui deviennent considérables à l'époque des fortes crues.

A la période d'étiage, on abaisse les vannes jusqu'à ce que. le niveau d'eau ait atteint la hauteur des grandes eaux. La prise d'eau est constituée par une galerie à deux orifices, à travers lesquels l'eau s'écoule dans un canal d'amenée à ciel ouvert de I.900 mètres de longueur, pour passer ensuite dans un tunnel de 2.900 mètres en partie maçonné et en partie ménagé dans les rochers. La conduite libre, à la faible pente de I $\%$, aboutit à son extrémité aval, au bassin d'eau de Morbegno.

A goo mètres de la tête amont du canal, on a ménagé un premier déversoir pour évacuer les sables et les cailloux déposés par les eaux. A la tête aval, il y a un second déversoir destiné à l'écoulement des eaux emmagasinées pendant la période de faible charge de l'usine. Chaque déversoir peut faire écouler $25^{\mathrm{m} 3}$ d'eau par seconde. Un troisième déversoir se trouve situé entre le dernier et avant-dernier tunnel. Dans le dernier tunnel le canal s'élargit, formant une chambre de décantation de 7 sur 25 mètres, ayant 5 mètres de profondeur. La vitesse de l'eau y est réduite de 
2, 5 mètres à 0,7 mètre par seconde. Les apports des eaux s'y arrêtent et peuvent être évacués à travers des vannes de chasse ménagées dans la paroi de la chambre de décantation.

Cette chambre d'eau est le point de départ de la conduite forcée, composée de deux tuyaux de 68 mètres de longueur et $2^{\mathrm{m}} 50$ de diamètre, inclinés à $45^{\circ}$ et aboutissant à leurs extrémités aval à la station centrale. Les tuyaux sont composés de feuilles en tôle d'acier, assemblés au moyen de rivets.

Chaque conduite se partage, dans la salle des machines, en deux parties, dont chacune aboutit à une turbine de 2.000 chevaux effectifs.

Les eaux de décharge sont restitućes à l'Adda par une galerie de roo mètres de longueur et 20 mètres de largeur. Le projet hydraulique a été imaginé par la maison Ganz, tandis que l'élaboration des plans détaillés et la surveillance de la construction ont été confiées à M. Gianfranceschi, ingénieur à Milan.

La station centrale est disposée pour quatre groupes de turbines et alternateurs; les premiers trois groupes de 2.000 chevaux chacun sont déjà installés, tandis que l'établissement d'un quatrième groupe de 3.000 ou 4.000 chevaux est prévu.

Les turbines à réaction du type Francis, de i 50 tours par minute, ont été fournies par la maison Ganz et $\mathrm{Co}$, de Budapest.

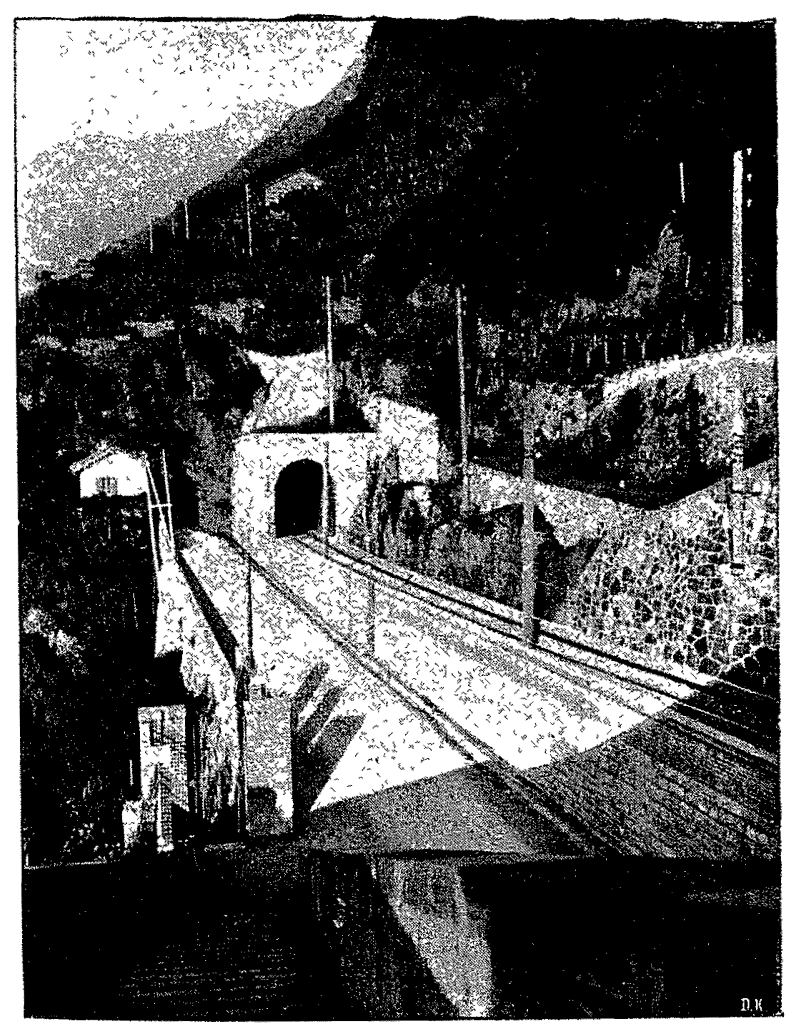

Fig. Ir. - Passage de la ligne primaire au-dessus d'un tunnel.

La chute totale, c'est-à-dire la différence des niveaux entre l'eau dans le bassin de distribution et dans le bassin collecteur de décharge, est 30 mètres, laquelle cependant est réduite à 27 et même 26 mètres, par suite de l'élévation du niveau aval dans la période des grandes eaux.

Chaque turbine peut être indépendamment mise hors de service par une vanne d'arrêt, ménagée dans l'embranchement du tuyau d'amenée.

Les dynamos sont directement accouplées aux turbines, de sorte qu'un groupe électrogène ne possède que deux paliers. Ceux-ci sont à graissage automatique à bagues; ils sont refroidis par l'eau. La partie mobile d'une turbine

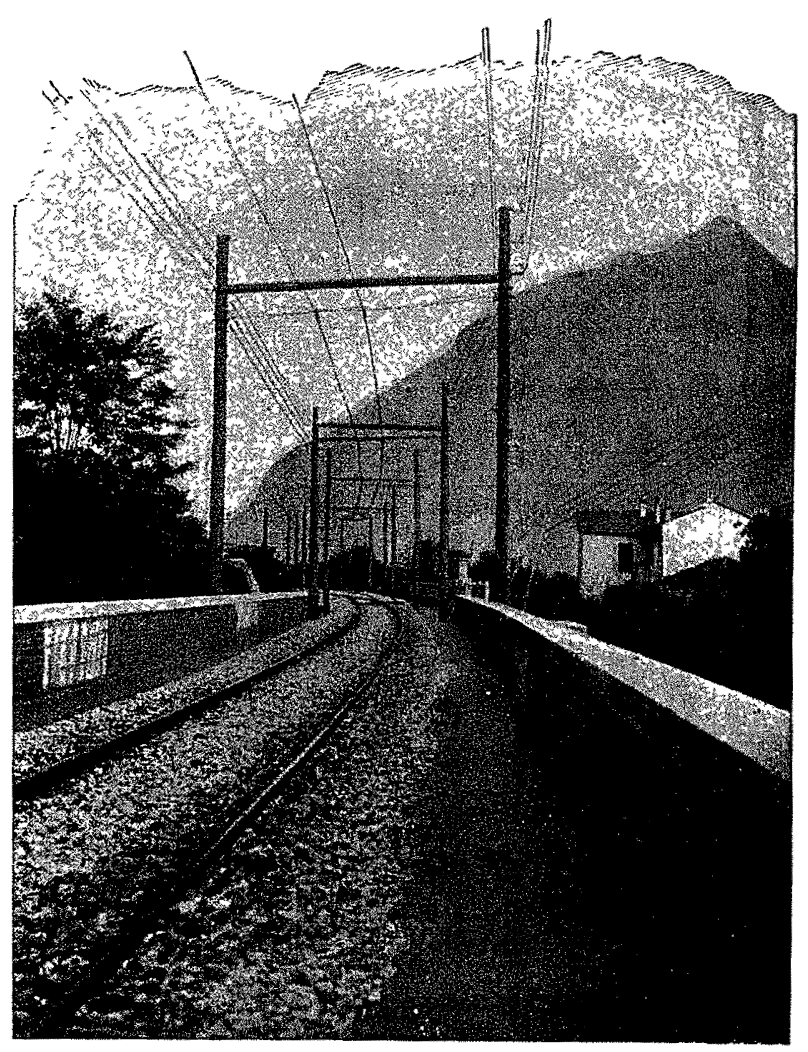

F1g. I2. - Vue de la voie et des lignes primaire et secondaire.

consiste dans un disque en acier, sur lequel est vissée la couronne, dont chaque partie est facilement rechangeable.

L'admission de l'eau est gouvernée par des ailes réglables du genre Fink. Le réglage peut être effectué à la main ou par un régulateur centrifuge du tableau même de distribution par l'intermédiaire d'une chaîne.

A la période des fortes crues, l'Adda porte beaucoup d'impuretés, de sorte que l'épuration aurait été très difficile. Par suite, le servo-moteur des turbines est actionné par de l'huile comprimée à ro atmosphères. Chaque turbine actionne une pompe qui comprime l'huile dans un accumulateur commun aux trois groupes.

Le régulateur Ganz consiste, en principe, en un pendule centrifuge du type Hartung, lequel actionne le piston du distributeur de l'huile. Ce distributeur conduit l'huile comprimée aux deux cylindres du servo-moteur, ou la laisse sortir suivant le cas.

Le servo-moteur déplace directement les aubes directrices; toute variation de la vitesse de la turbine, ayant pour conséquence une variation de l'appareil centrifuge, est immédiatement suivie d'une variation de la pression entre les deux cylindres du servo-moteur, faisant ainsi augmenter ou diminuer l'admission de l'eau. L'huile sortant du servomoteur est de nouveau pressée dans l'accumulateur par les pompes à huile.

Les génératrices, dont la fourniture a été confiée pour des raisons financières à la maison Schuckert et $C^{\text {le }}$, de 
Nuremberg, produisent le courant à 20.000 volts et à 15 périodes. Elles consomment 1.560 chevaux, à la charge normale de ro5o kilowatts sur un circuit extérieurayant un facteur de puissance de 0,7 .

Elles peuvent répondre à des surcharges temporaires de 2000 chevaux (avec $\cos \varphi=0,7$ ). L'élévation de température de l'enroulement n'excède pas $45^{\circ}$ centigrades audessus de la température ambiante, après une charge continue de 1.500 chevaux. La baisse de tension entre marche à vide et pleine charge, ne dépasse pas i $5 \%$, tandis qu'en enlevant momentanément toute la charge, la tension ne croit que de $10 \%$.

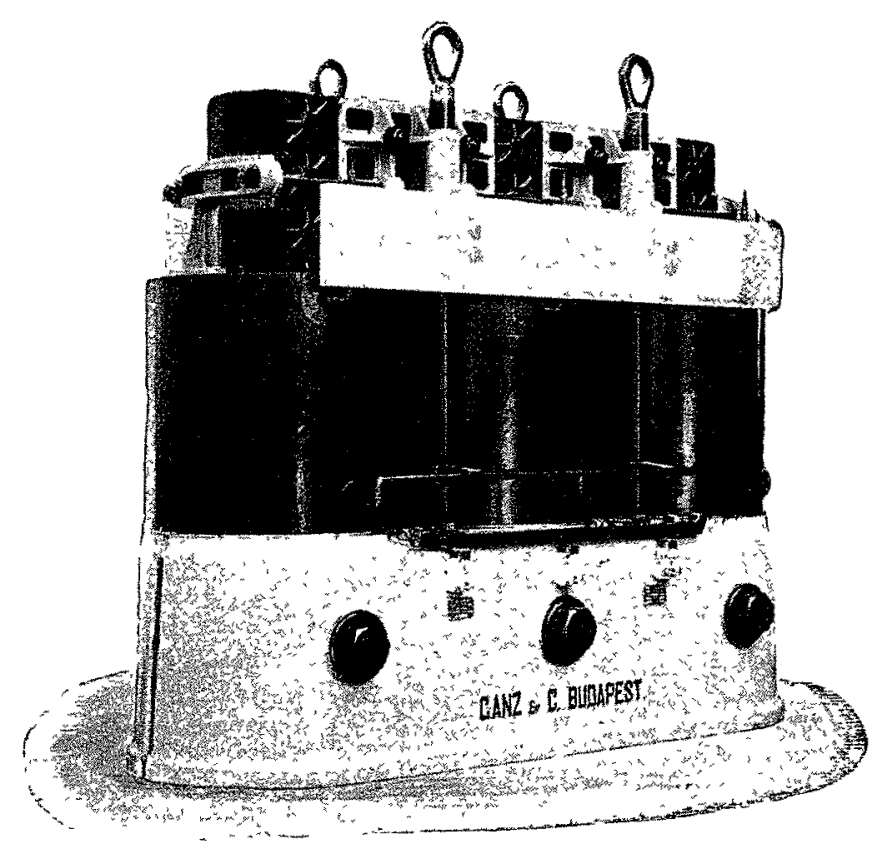

Fig. 13. - Transformateur des sous-stations.

En court-circuit, la génératrice supporte, parait-il, six fois l'intensité normale du courant ; un court-circuit de 120 " ne cause aucune détérioration de l'isolement. Les génératrices peuvent produire, sans aucun inconvénient, des courants à 30.000 volts, pendant une demi-heure; mais pour éviter l'élévation excessive de la tension, qui pourrait avoir lieu éventuellement lors d'un mauvais fonctionnement du régulateur de la turbine, l'excitatrice qui est directement calée sur l'arbre commun, insère automatiquement de la résistance dans le circuit d'excitation, lors d'un emballement de la machine, de manière que la tension des génératrices ne puisse dépasser 25.000 volts, même si le nombre de tours augmente à 250 . Cette précaution a été surtout nécessaire pour protéger non pas les dynamos, mais bien les appareils de mesure du tableau de distribution.

Le poids total d'une génératrice monte à 69.300 kil.; la partie mobile, l'arbre compris, pèse $43.800 \mathrm{kil}$.

Le tableau de distribution est divisé en six panneaux, dont quatre pour les groupes électrogènes, et deux pour deux lignes externes.

L'une des deux lignes externes sert provisoirement pour les charges d'essai de dynamos; l'autre suffit seule pour le trafic d'aujourd'hui. Les dynamos sont raccordées aux barres collectrices du tableau de distribution par des conducteurs bien isolés placés sous le sol.

Tous les instruments de mesure sont reliés aux conduc- lìg. I4. - Schéma des connexions d'une sous-station. $J$, Isolateur de section. $-S$, Rails. $-L$, Plaques connectées à la terre.

Circull d 20.000 volts .

$A$, Coupe-circuits fusbles $B$, lnterrupteur.

$C$, Bobines de self

$l$, Disques en zinc

E. Résistances en charbon.

$F$, Cornes type Sientens.

$G$, Prmalre du tranoformatemr.

Cirenil à 3000 volls :

a, Coupe-enrcuts fusioles.

$b$, Interrupteur.

$c$, Bobines de self.

$d$, Disques en zınc

$e$, Résistances en charbon.

f, Cornes type Siemens.

g. Secondative da transformateur.

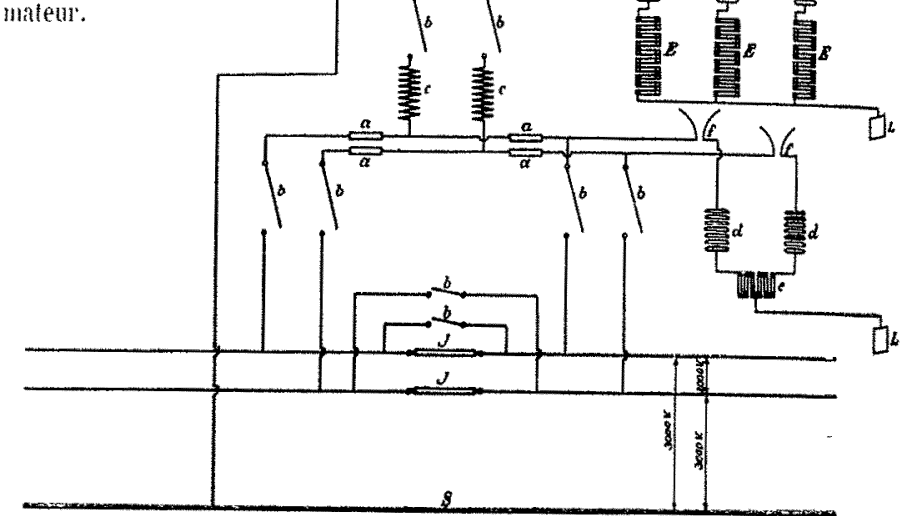

teurs principaux au moyen de transformateurs-réducteurs, de sorte qu'il est impossible que le personnel chargé de la manipulation du tableau prenne accidentellement contact de la haute tension.

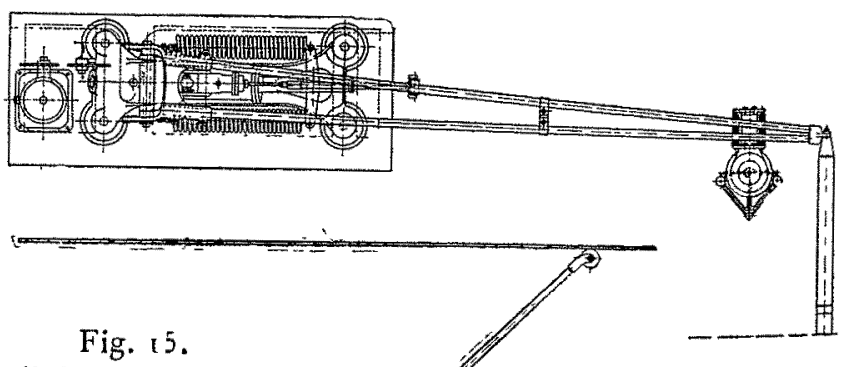

Appareil de prise de courant.

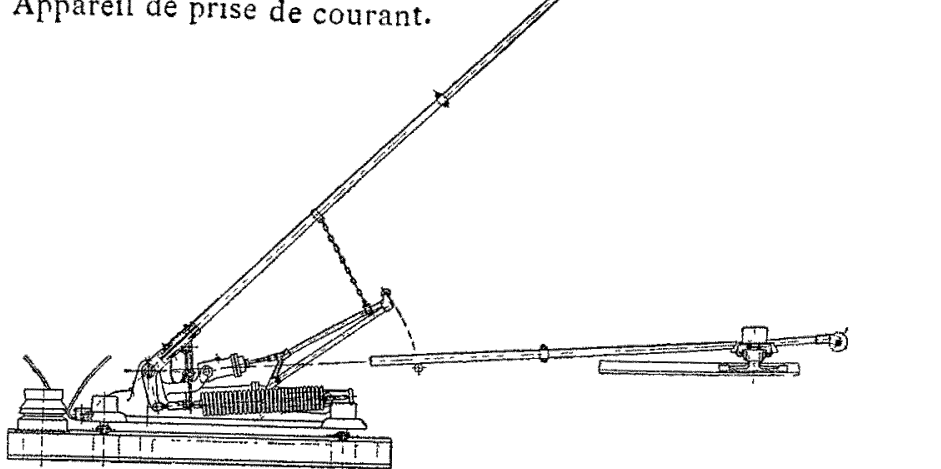

Les dynamos sont protégées contre des surcharges par des coupes-circuits fusibles montés dans des tuyaux en porcelaine.

Les interrupteurs primaires des dynamos sont maniables du devant du tableau, tandis que les interrupteurs des 
lignes externes sont manœuvrés d'un local derrière le tableau de distribution.

$2^{\circ}$ Conducteurs. - Le courant primaire à 20.000 volts triphasés est amené par la ligne à haute tension aux sousstations de transformateurs. Les poteaux sont en mélèze de Carinthie, ayant un diamètre supérieur minimum de 20 centimètres. Avant le plantage, ils ont été carbonisés et goudronnés à la partic inférieure. Aux stations ils sont placés dans des socles en fonte.

l.es conducteurs primaires sont fixés sur des isolateurs spéciaux situés sur le prolongement des poteaux des
Les fils dè contact sont amenés à 6 mètres au-dessus des rails. Aux tunnels, cette hauteur diminue jusqu'à 4 mètres 80 . Les fils de contact sont élastiquement suspendus par deux isolateurs spéciaux en ambroine, chacun maintenu par un fil tendeur d'acier, attaché aux isolateurs en porcelaine. L'isolateur d'ambroine se termine par deux boutons, sur lesquels sont attachées une paire de joues en fer étamé, qui embrassent le fil de contact. Celui-ci pouvant se déplacer dans le sens vertical autour d'un axe horizontal, n'est soumis à aucun effort de flexion au point de suspension, comme c'est le cas des suspensions rigides.

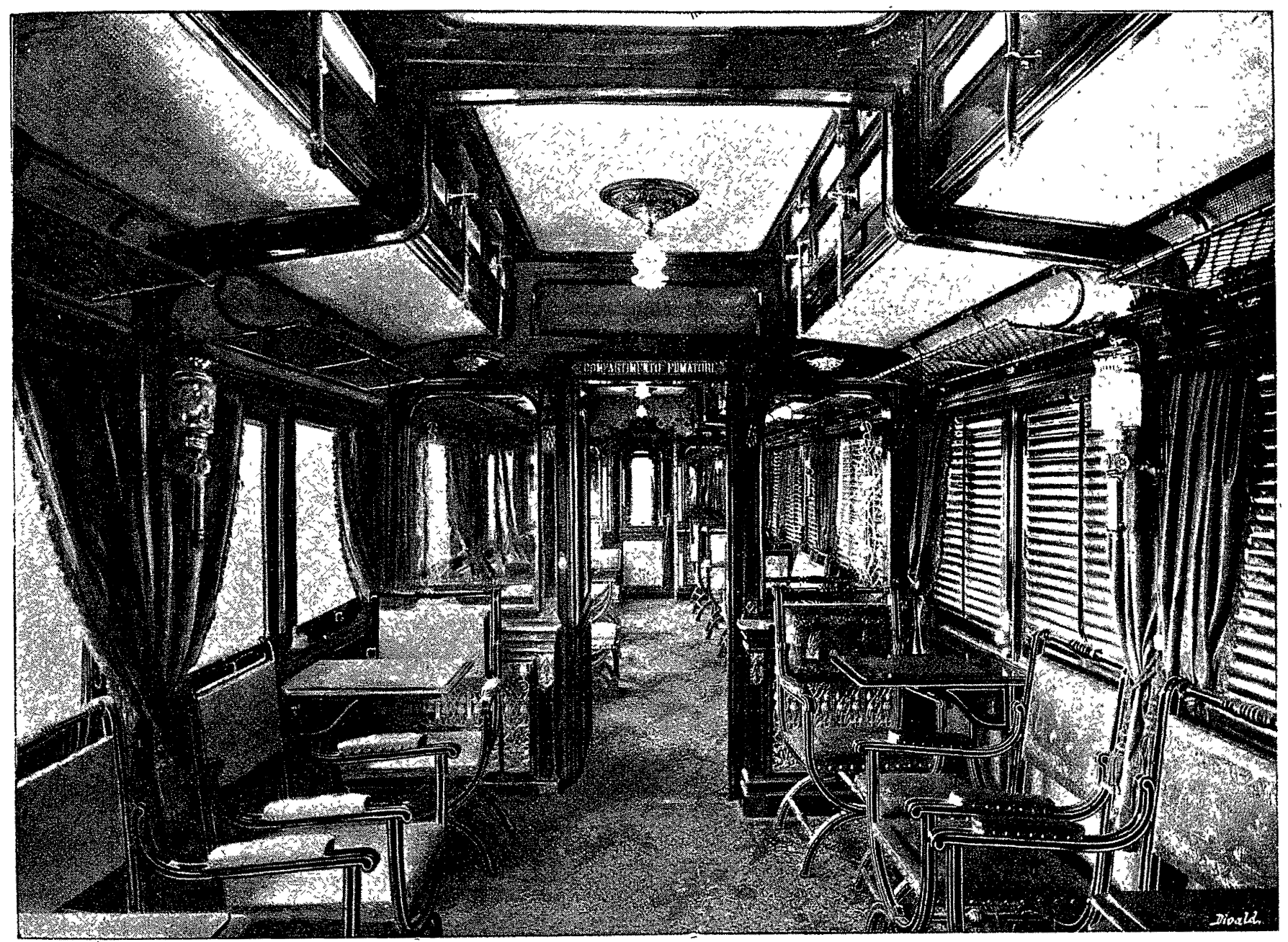

Fig. I6. - Vue intérieure d'une voiture-salon (Compartiment des fumeurs).

conducteurs de contact. Aux entrées des tunnels cependant la ligne primaire quitte la ligne de contact et passe audessus des tunnels sur des poteaux distincts, jusqu'à la sortie.

Les sous-stations divisent la ligne primaire par leurs interrupteurs à 20.000 volts en autant de sections indépendantes. Les tronçons de Morbegno-Castione et ColicoChiavenna ont, en outre, des interrupteurs distincts.

La ligne de contact est constituée par deux fils de cuivre étiré de 8 millimètres de diamètré, le troisième conducteur étant remplacé par les rails, lesquels reçoivent aux éclissages des joints en cuivre de 6 millimètres de diamètre.
Pour empêcher que l'appareil de prise de courant n'occasionne un court-circuit entre les fils de contact à différents potentiels aux aiguillages ou aux croisements aériens, ces fils sont placés à des hauteurs différentes, obligeant ainsi les rouleaux de contact à prendre une position oblique.

En alignement droit et en courbures dont le rayon dépasse 1000 mètres, les fils de contact sont supportés par des poteaux à potences; dans les stations par des poteaux à doubles potences. Dans les courbures plus petites, deux poteaux réunis à la partie supérieure par des traverses supportent les fils. 
A l'entrée et à la sortie de toutes les stations, un tronçon de la ligne de contact, d'une longueur de 300 mètres, se trouve isolé du reste de la ligne par des isolateurs de section. Ces tronçons peuvent être mis sous tension par des interrupteurs spéciaux placés aux stations.
$3^{0}$ Sous-stations. - Il y a 9 sous-stations de transformation réparties le long de la ligne; chacune d'elles contient un transformateur triphasé d'une capacité de 300 kilovoltampères, sauf la dernière, à Abbadia, où deux transformateurs pareils sont installés.

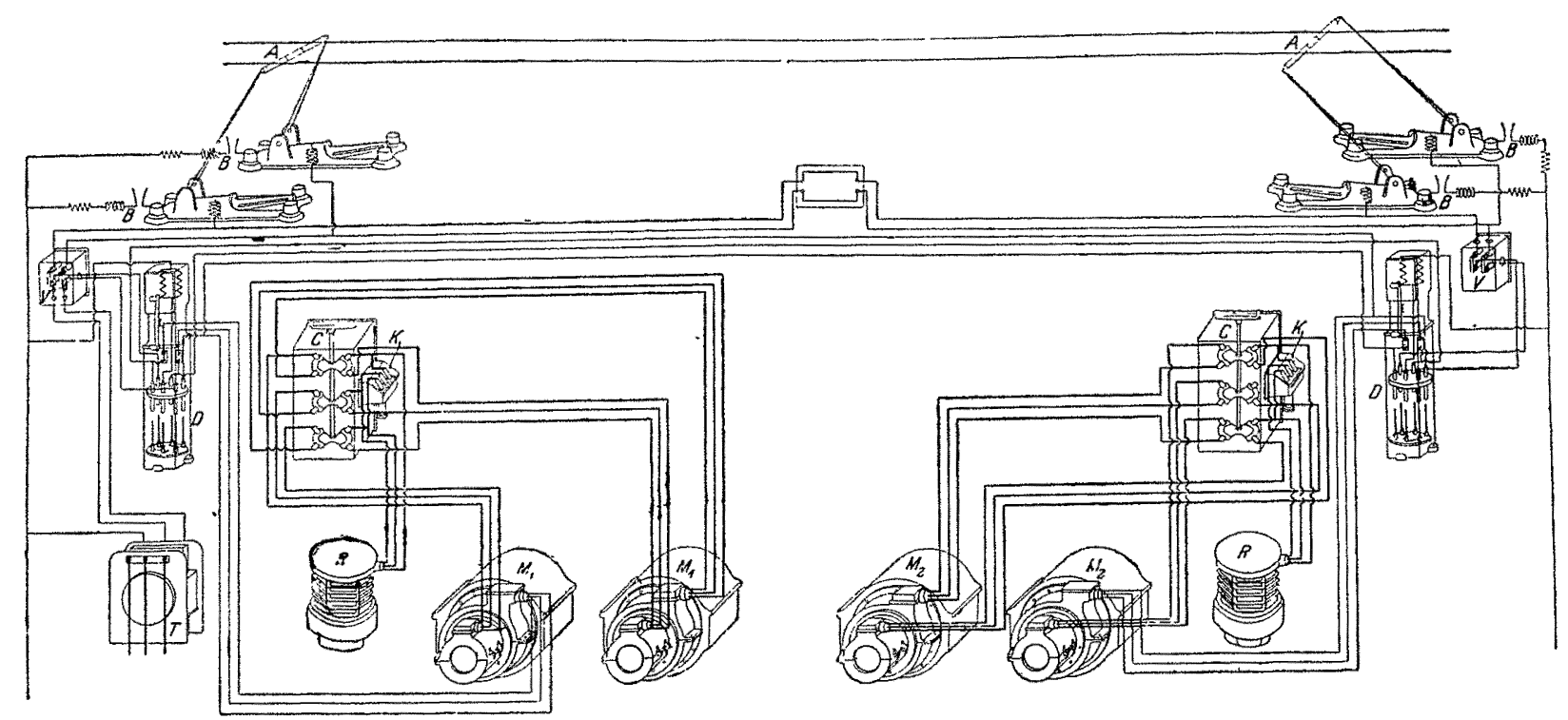

Fig. I7. - Schéma des connexions d'une voiture motrice.

1 , Appareils de prise de courant. $-B$, Parafoudres. $-C$, Contrôleur, $-D$, Interrupteurs primaires. - $K$, Appareils de court-cırcuits, $M_{1} M_{2}$, Moteurs à haute tension. - $M_{1}^{\prime} M_{2}^{\prime}$, Moteurs à basse tension. - $R$, Rhéostats liquides. $-T$, Transformateur pour les circuits d'éclcirage, chauffage, moteurs compresseurs. - $T$, Caisse de distribution avec coupe-circuits fusibles.

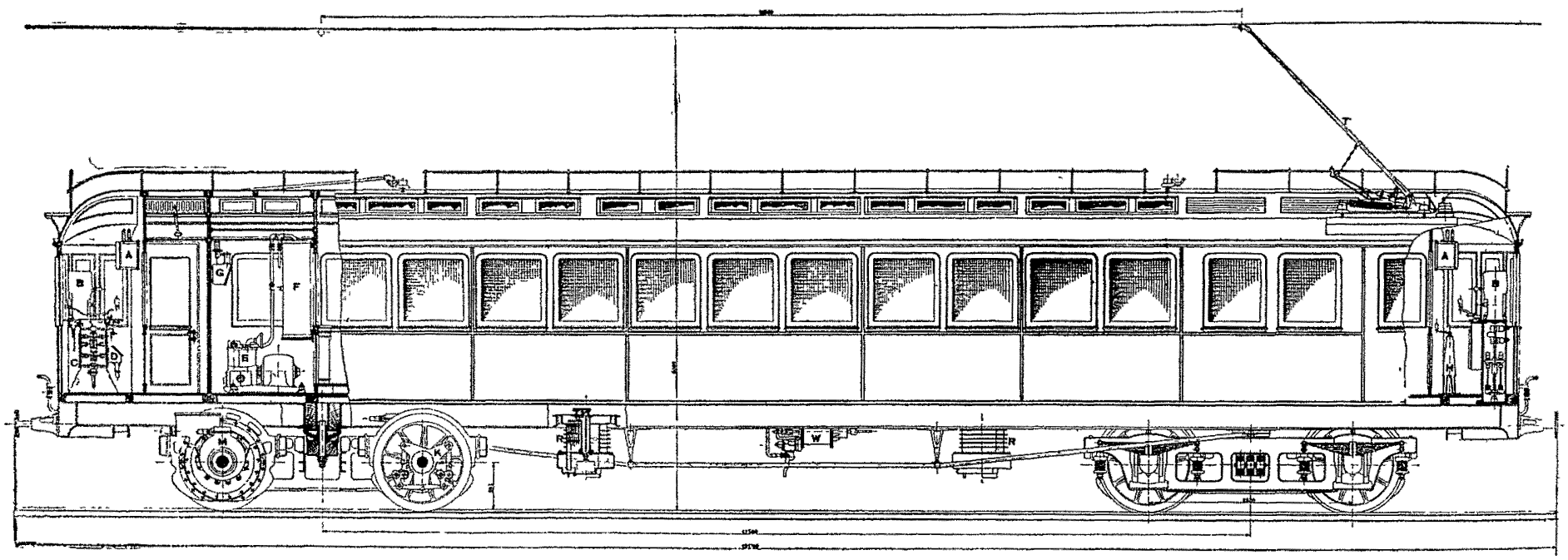

Fig. 18, - Vue et détails d'une voiture automotrice.

Ces interrupteurs ne peuvent être fermés que lorsque le sémaphore est dans la position "libre ». Les lignes de contact dans la station même peuvent recevoir de la tension indépendamment des tronçons isolés, par un troisième interrupteur.

Toute la ligne de contact forme une ligne ininterrompue le long de la voie, car avant les stations la ligne de contact se partage en deux parties dont l'une, faisant un détour derrière la station, rejoint la ligne principale à la sortie après le dernier isolateur de section.

Des paratonnerres à cornes, placés sur les poteaux de la ligne secondaire, protègent tant la ligne primaire que la ligne secondaire contre les décharges atmosphériques. Les résistances ohmiques de ces paratonnerres sont aussi portées par les poteaux.
Une cloison verticale sépare le transformateur de tout l'appareillage de la maison, c'est-à-dire des coupe-circuits fusibles, des interrupteurs et du ventilateur électrique destiné à refroidir le transformateur. Le moteur du ventilateur est alimenté par une bobine secondaire distincte, donnant une tension de 14 volts.

Le rapport de transformation des transformateurs est de 6 à I ; leur capacité normale est 30 o kilovoltampères, mais ils peuvent supporter une surcharge momentanée de quatre à cinq fois la charge normale.

La figure 14 représente le schéma d'installation d'une sous-station. Nous y voyons, outre les appareils décrits, un interrupteur dans la ligne primaire, destiné à séparer le transformateur, en cas de besoin, du reste de cette ligne. A proximité de chaque sous-station, la ligne de contact 
se trouve divisée par des isolateurs en sections indépendantes. Le transformateur peut être connecté à chacune de ces sections au moyen d'interrupteurs distincts. La ligne primaire est protégée, dans chacune de ses trois branches, par des coupe-circuits fusibles, tandis que la ligne secondaire n'en reçoit que dans les deux feeders des fils de contact, la troisième branche étant directement connectée aux rails.

Cette installation permet, en marche normale, la mise en parallèle de toutes les sous-stations et, en cas d'accident, la mise hors circuit d'une section quelconque.

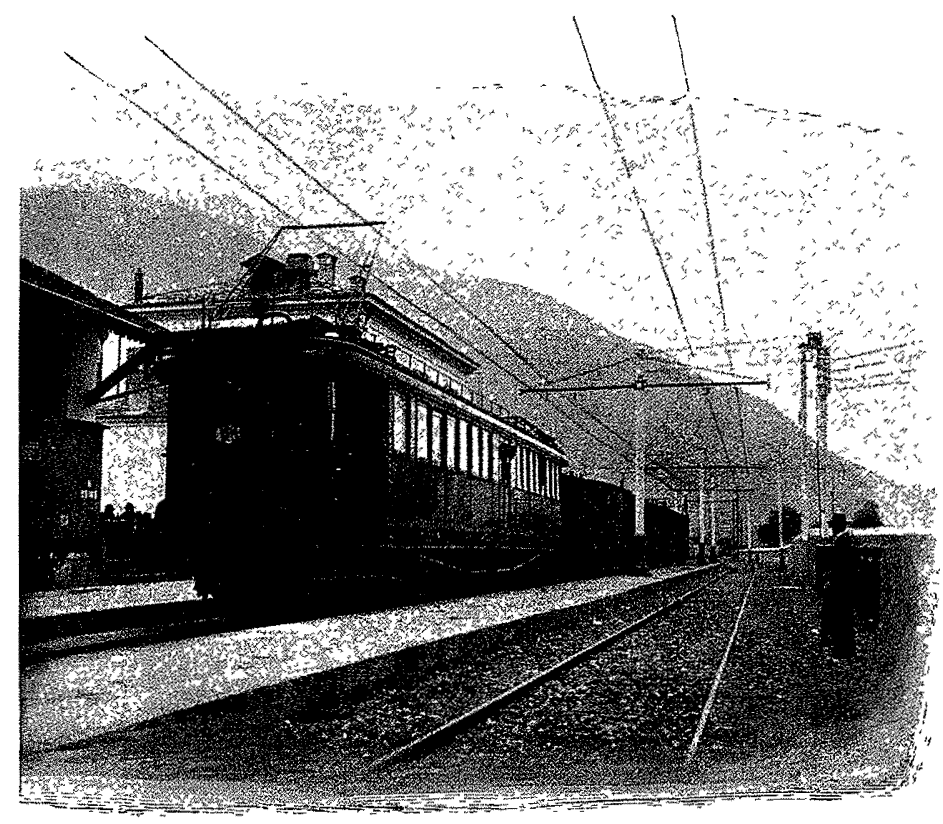

Fig. 19. - Train rapide.

40 Matériel roulant. - Le trains de voyageurs sont remorqués par des voitures motrices, les trains de marchandises par des locomotives. Ces dernières peuvent remorquer des trains de marchandises de 400 tonnes à la vitesse de 30 à 35 kilomètres ou bien des trains de voyageurs de 250 tonnes à 60 à 70 kilomètres à l'heure.

Voitures motrices. - Les voitures motrices ressemblent aux voitures ordinaires à deux boggies, chacun des deux essieux d'une boggie portant directement son moteur. Chaque extrémité d'une voiture contient une cabine fermée pour le conducteur.

Le poids total d'une voiture motrice, y compris l'équipement électrique, monte à 53 tonnes. Une voiture traîne, outre son poids propre, 5 à 7 voitures ordinaires de $\mathrm{lo}$ à $\mathrm{r} 2$ tonnes, à une vitesse de 65 kilomètres à l'heure.

L'appareil de prise de courant consiste, en principe, en deux rouleaux de cuivre de 650 millimètres de long sur 80 millim. de diamètre, isolés par une cale de bois faisant partie de l'axe de rotation. Les tiges supportant ces rouleaux peuvent pivoter autour d'un axe horizontal porté par le toit de la voiture, l'élévation et l'abaissement du trolley étant provoqué par un piston à air comprimé.

Chaque essieu porte un moteur électrique dont la partie fixe est rigidement attachée à la carcasse et dont la partie tournante actionne directement les roues par l'intermédiaire d'un accouplement élastique. Les coussinets de l'induit sont supportés par la carcasse de l'inducteur, de sorte que tout le poids du moteur, quoi qu'il soit directement monté sur l'essieu, repose sur des ressorts.

Chaque boggie porte un moteur à haute tension et un à basse tension. Le rapport de transformation entre inducteur et induit est de I : Io; la tension au rotor ne dépasse donc jamais 300 volts. Le démarrage et le réglage de la vitesse se font exclusivement dans un circuit à basse tension.

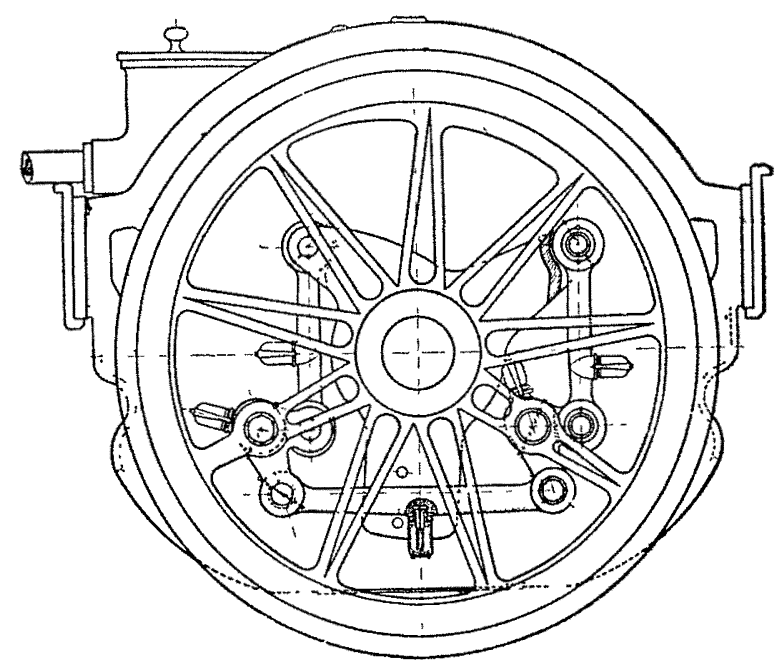

Fig. 20. - Vue de l'accouplement du motcur.

En couplant deux moteurs à champ tournant en " cascade ", c'est-à-dire, en connectant le rotor de l'un au stator de l'autre, on réduit alors la vitesse de marche à la moitié de sa valeur initiale, si les mouvements des deux rotors sont invariablement liés.

Ce couplage est obtenu au moyen de deux contrôleurs situés dans les deux cabines; leurs mouvements sont rendus solidaires par l'intermédiaire d'une chaîne. La manivelle du contrôleur n'a que trois positions. Dans la première, les moteurs sont hors circuit, dans la seconde, ils sont couplés en cascade, dans la troisic̀me, les moteurs à haute tension seuls sont intercalés.

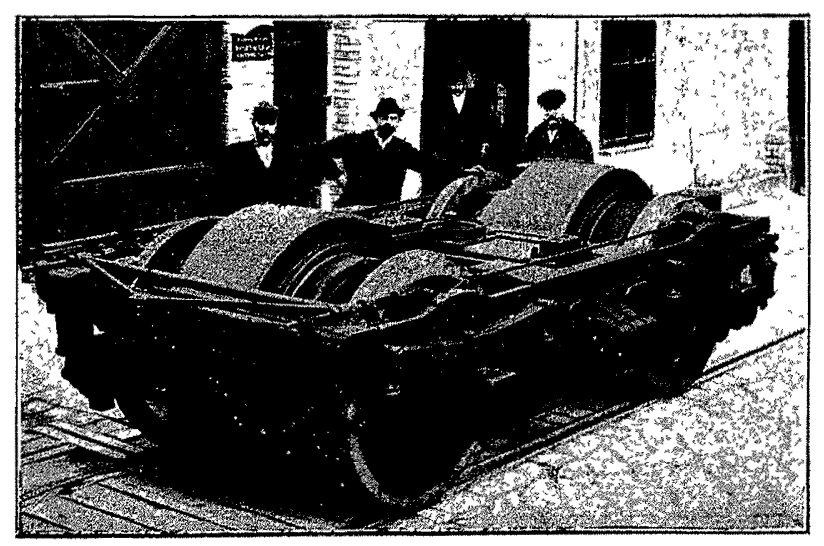

Fig. 21. - Boggie d'une voiture motrice.

Au moment du démarrage, une résistance liquide est intercalée dans le circuit de l'induit des moteurs. Cette résistance consiste en un récipient en fonte dans lequel trois groupes de tôles d'inégales longueurs sont plongés. Le circuit de l'induit est connecté à ces trois groupes.

Une pression pneumatique fait monter dans ce récipient une solution alcaline. Tant que le liquide ne touche pas les 
tôles, le.circuit induit est ouvert et le moteur ne développe aucun couple; lorsque le liquide ferme le circuit des trois phases, le moteur démarre. A mesure que l'eau monte, la résistance intercalée diminue, et la vitesse du moteur augmente. Lorsque le liquide atteint la hauteur maxima, un appareil met automatiquement en court-circuit l'induit du moteur.

Avant le démarrage, la manivelle du contrôleur est mise dans la position : "petite vitesse " correspondant au cou-
L'insertion des moteurs dans le circuit, l'élévation du liquide dans les rhéostats, la régularisation de la vitesse, les mouvements de l'appareil de prise de courant, se font exclusivement au moyen de l'air comprimé. Un compresseur, actionné par un petit moteur triphasé, comprime l'air dans les réservoirs. Ces derniers ont chacun deux chambres réunies par une soupape de retenue qui permet la communication de l'air de la première dans la seconde, mais non pas en sens inverse. La seconde chambre contient l'air

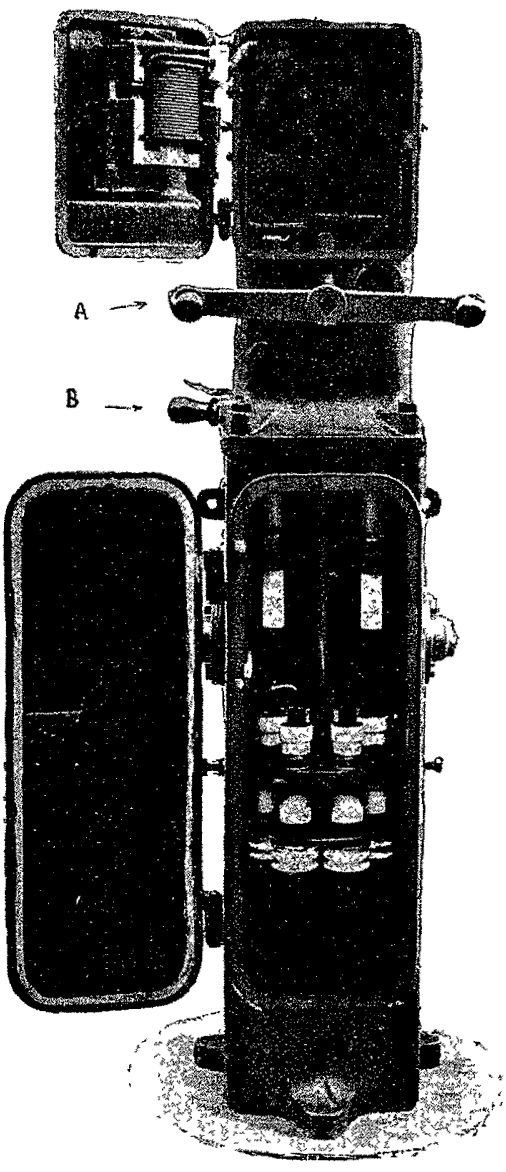

Fig. 22. - Vue de l'interrupteur primaire ouvert.

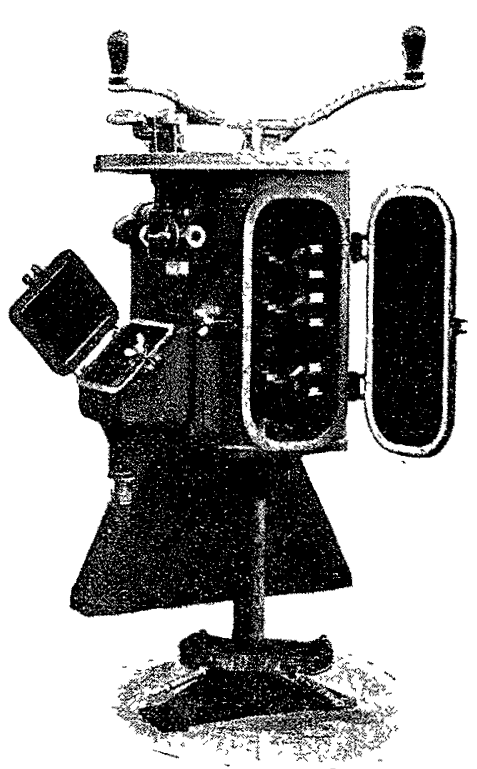

Fig. 23. - Vue d'un contrôleur.

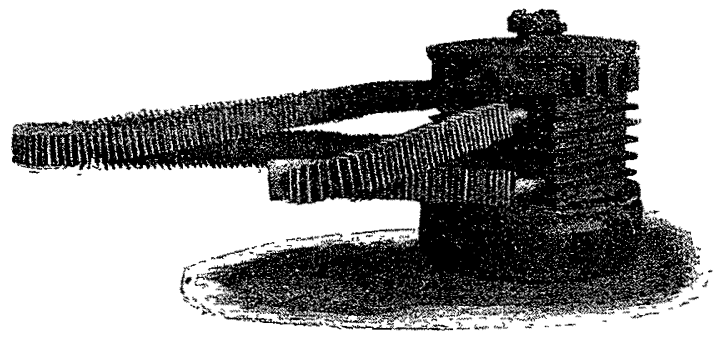

Fig. 24. - Rhéostat liquide d'une voiture motrice.

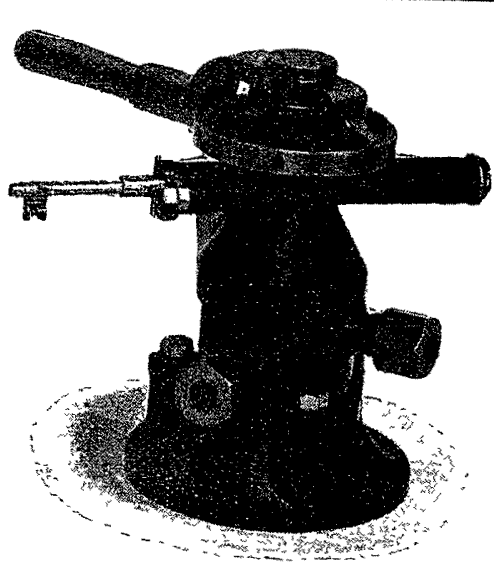

Fig. 25. - Appareil commandant la manœuvre des trolleys.

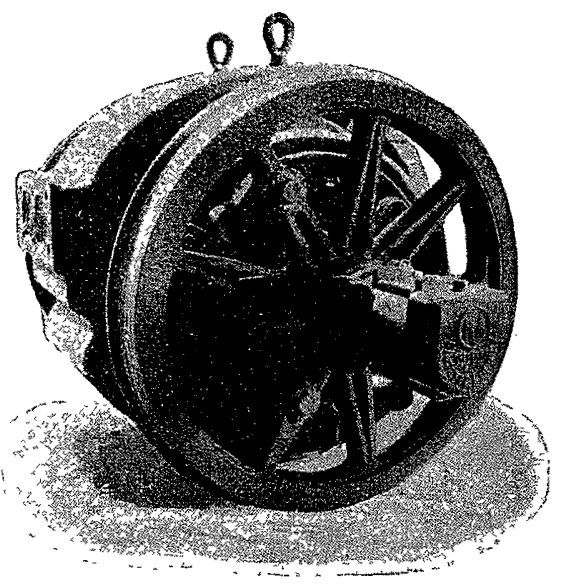

F1g. 26. - Vue latérale du moteur avec paire de roues. plage des moteurs en cascade; on ouvre ensuite la soupape à air de l'interruption primaire qui ferme le circuit de 3.000 volts dans les inducteurs. Il suffit alors de faire arriver le liquide dans le rhéostat pour que les moteurs atteignent la vitesse de régime du couplage en cascade.

Pour passer à la grande vitesse, on coupe d'abord le courant à 3.000 volts et on met hors circuit les moteurs à basse tension; on recommence alors la manœuvre précédente.

Locomotives. - Les locomotives, à quatre essieux, sont composées de deux parties symétriques, réunies par une jonction mobile. Chaque essieu porte un moteur à haute tension de 150 chevaux normaux. Tous les quatre moteurs sont à haute tension; ils peuvent être séparément mis sous tension ou déconnectés du circuit par un contrôleur spécial, suivant l'effort demandé à la locomotive. Ce contrôleur est situé dans le milieu de la cabine du conducteur, tandis que les quatre rhéostats liquides sont placés dans les deux rebords de la locomotive. comprimé pour le frein Westinghouse, de sorte que, s'il y avait un accident dans l'installation pneumatique de la loco. motive, on pourrait encore l'arrêter par le frein Westinghouse. Le contrôleur permet la régularisation parfaite de la vitesse en laissant entrer une plus ou moins grande quantité d'eau dans les rhéostats. Le poids propre de la locomotive est de 46 tonnes. Son effort de traction maxima, mesuré à la jante des roues motrices est de 8.000 kilogs à 30 kilomètres à l'heure. La charge maxima remorquée est de 450 tonnes sur une rampe de io $\%$.

Inspection de la ligne, traction, service du trafic. - Le service de la station centrale dure pendant 18 à 20 heures par jour. Le personnel est divisé en trois groupes, dont chacun comporte un électricien, un mécanicien et un graisseur. L'inspection du service est confiée au mécanicien en chef et à son second, de sorte qu'il y a en tout onze employés. Quatre gardiens sont en outre affectés au service des vannes du canal. 
Le personnel chargé de la surveillance de la ligne entière se compose de trois monteurs et d'un ouvrier par sousstation de transformateurs. Tous les gardes-voie, qui sont en même temps chargés de la surveillance de la ligne aérienne, sont munis de cahiers spéciaux sur les feuilles perforées desquels se trouvent imprimés tous les défauts possibles de la ligne.

Aussitôt qu'un garde-voie aperçoit un défaut quelconque sur la ligne, il l'inscrit à la place convenable sur la feuille imprimée, qu'il déchire et qu'il envoie de suite à la station voisine. Le chef de station télégraphie au monteur en chef qui envoie le personnel nécessaire. Si ce défaut pouvait endommager l'appareil de prise de courant, le conducteur du train en route serait également averti et il passerait le point dangereux avec la prise de courant abaissée. On a vil, en effet, que l'on pouvait facilement mancuvrer cet appareil pendant la marche.

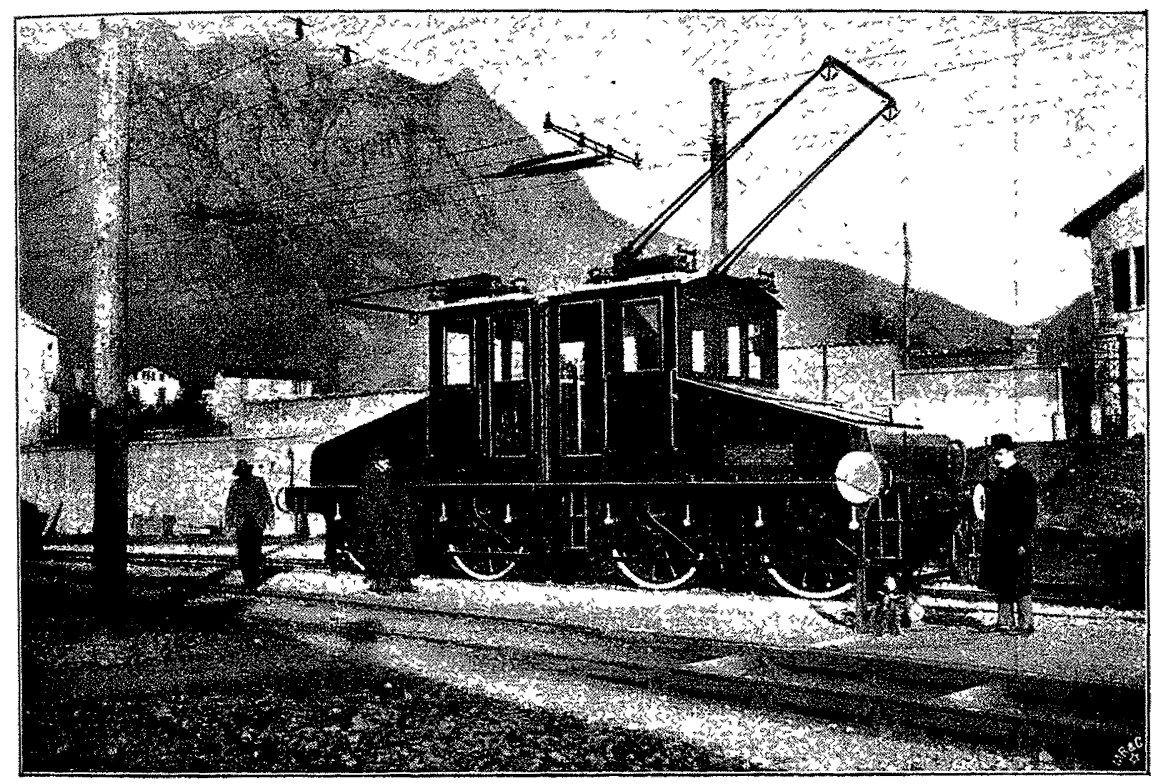

Fig. 27. - Vue générale de la locomotive électrique.

Le service sur la voiture motrice ou sur la locomotive n'occupe qu'une personne. Le chef de train cependant connaît aussi entièrement le service du conducteur, et inversement; ces deux employés font alternativement les deux services, de sorte qu'il y a toujours sur le train deux personnes connaissant parfaitement la manœuvre du véhicule moteur.

Avec l'ouverture du service électrique, on a en même temps complètement séparé le trafic à longues distances du trafic local, et le trafic des voyageurs de celui des marchandises. Les tarifs des trains express (à ${ }^{r}$ re et $2^{e}$ cl.) n'ont pas changé, tandis que ceux des trains locaux (à $2^{\theta}$ et $3^{\circ}$ classes) ont été réduits.

Le service électrique a été officiellement ouvert, le 4 septembre 1902, sur les parties Colico-Chiavenna, et ColicoSondrio, et le 5 octobre 1902 , sur la partie plus difficile de Lecco à Colico. Depuis lors, le système fonctionne sans défaut à la satisfaction complète de la Compagnie du chemin de fer, et du public voyageur.

\section{J. Courbier,}

Ancien Elève de l'Ecole Polytechnique, Ingénieur-Electricien.

\section{SUR LA COURBE DES DÉBITS D'UNE SOURCE (1)}

( Considérons une source issue d'une nappe souterraine (terrains perméables), et des périodes $P$ où les pluies ne profitent pas sensiblement à cette nappe, conformément à la loi que Dausse a indiquée pour le bassin de la Seine (énoncé de Belgrand, La Seine, Etudes hydrologiques, Paris, I 872 , p. 65). Admettons que, dans ces périodes, un régime tende à s'établir de façon qu'à chaque valeur du débit $Q$ de la source corresponde une valeur unique du volume $V$ d'eau qui y est contenu, $V$ étant fonction croissante de $Q$ : l'équation de continuité $d V=-Q d t$, avec $V=f(Q)$, conduit i la relation :
(1)

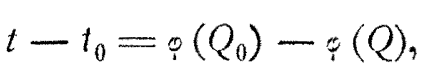
$\varphi^{\prime}(Q)=\frac{f^{\prime}(Q)}{Q}$

"Le régime en question, s'il existe, sera dit, le régime propre ou non influencé de la source.

" Avec deux axes rectangulaires $O Q_{0}$ (abscisses), $O Q$ ordonnées, construisons, d'après les résultats de l'expérience, les courbes $t-t_{0}=$ const. Au moyen du graphique obtenu, quand nous serons dans une période $P$, connaissant $Q_{0}$ au temps $t_{0}$, nous pourrons prévoir à l'avance le débit $Q$. (1) peut encore s'écrire:

(2) $t+\stackrel{\varphi}{(Q)}=t_{0}+\varphi_{i}\left(Q_{0}\right)=\tau=$ const.,

- étant une constance spécifique de la source. Prenant deux axes rectangulaires $O_{1} t$ (abscisses), $O_{1} Q$ (ordonnées), (2) représente une courbe, que nous pourrons encore construire d'après les résultats de l'expérience, et que nous appelons la courbe des débits de la source (dans les périodes de régime propre). $Q$ est fonction décroissante du temps $t$.

"Si les périodes $P$ sont assez longues, les prévisions faites à l'aide de (1) ou (2) seront des prévisions à longue échéance.

"Le graphique (1), plus commode à construire que la courbe (2), a des propriétés intéressantes qui peuvent en faciliter la construction. Supposons que nous attribuions à $t-t_{0}$ des valeurs en progression arithmétique: les courbes $t-t_{0}=\mathrm{I}, 2,3, \ldots$ se déduisent de la courbe $t-t_{0}=1$ par une construction géométrique simple. Elles sont toutes comprises pratiquement entre la bissectrice de $Q O Q_{0}$ et l'axe $O Q_{0}$, dans le premier quadrant. La condition nécessaire et suffisante pour que le tarissement de la source ne se produise jamais est que toutes les courbes $t-t_{0}=$ const. passent par l'origine. Si la courbe $t-t_{0}=\mathrm{I}$ est concave ou convexe vers les $Q$ positifs, les autres courbes le sont en même temps. Quand ces courbes sont convexes, ou sont des droites passant par l'origine, la diminution du débit pendant l'unité de temps à partir du temps $t$ est d'autant plus grande que le débit au temps $t$ est plus grand.

( On peut construire le graphique (I) connaissant la

(I) Note de M. Edmond Maillet à l'Académie des Sciences, séance du 27 octobre 1903 . T. CXXXVII, no ${ }^{\circ} 7$. 